\title{
Diversity and Multiplexing Technologies by 3D Beams in Polarized Massive MIMO Systems
}

\author{
Xin $\mathrm{Su}^{1}$ and KyungHi Chang ${ }^{2}$ \\ ${ }^{1}$ College of Internet of Things (IOT) Engineering, Hohai University, Changzhou 213022, China \\ ${ }^{2}$ Electronic Engineering Department, Inha University, Incheon 402751, Republic of Korea \\ Correspondence should be addressed to KyungHi Chang; khchang@inha.ac.kr
}

Received 13 July 2015; Revised 14 November 2015; Accepted 29 November 2015

Academic Editor: Jing Liang

Copyright (c) $2016 \mathrm{X}$. Su and K. Chang. This is an open access article distributed under the Creative Commons Attribution License, which permits unrestricted use, distribution, and reproduction in any medium, provided the original work is properly cited.

\begin{abstract}
Massive multiple input, multiple output (M-MIMO) technologies have been proposed to scale up data rates reaching gigabits per second in the forthcoming $5 \mathrm{G}$ mobile communications systems. However, one of crucial constraints is a dimension in space to implement the M-MIMO. To cope with the space constraint and to utilize more flexibility in 3D beamforming (3D-BF), we propose antenna polarization in M-MIMO systems. In this paper, we design a polarized M-MIMO (PM-MIMO) system associated with 3D-BF applications, where the system architectures for diversity and multiplexing technologies achieved by polarized 3D beams are provided. Different from the conventional 3D-BF achieved by planar M-MIMO technology to control the downtilted beam in a vertical domain, the proposed PM-MIMO realizes 3D-BF via the linear combination of polarized beams. In addition, an effective array selection scheme is proposed to optimize the beam-width and to enhance system performance by the exploration of diversity and multiplexing gains; and a blind channel estimation (BCE) approach is also proposed to avoid pilot contamination in PMMIMO. Based on the Long Term Evolution-Advanced (LTE-A) specification, the simulation results finally confirm the validity of our proposals.
\end{abstract}

\section{Introduction}

Massive multiple input, multiple output (M-MIMO) is being developed as a promising technology for several attractive features [1-3]; for example, the system capacity can be theoretically increased by installing sufficient antennae, and the transmit power can potentially be reduced in inverse proportional to the square root of the number of applied antennae [4, 5]. This not only is relevant from a commercial standpoint but also provides green transmission to address health concerns in wireless communications [6]. When channel reciprocity is exploited in time division duplex (TDD) M-MIMO systems, the overhead related to channel compensation scales linearly only with the number of mobile user-equipment (UE) per cell rather than the number of antennae per base station (BS) [7]. Matched filtering is suggested as being optimal for linear precoders and detectors because thermal noise, interference, and channel estimation errors can theoretically vanish in $\mathrm{M}$ MIMO systems [1]. The remaining performance limitation is the pilot contamination [8], which is the residual interference caused by the reuse of pilot patterns. The above claims are validated based on several crucial but optimistic assumptions of perfect channel estimation, hardware implementations, and the number of antennae applied in practice. Recently, a lot of the literature has studied M-MIMO with more realistic assumptions [9-13].

On the other hand, there has been a gradual demand for the use of polarized antenna systems, especially for $5 \mathrm{G}$ mobile communications systems [14-16]. This is mainly because the antenna polarization is a pivotal resource to be exploited for the design of space-limited wireless devices. Techniques such as space-time diversity, multiplexing, and array processing can be applied to polarized antenna systems to boost system throughput.

In this paper, we propose a polarized M-MIMO (PMMIMO) array system, where three orthogonally colocated antenna branches are applied at each array element (AE) of an M-MIMO system. Three-dimensional beamforming (3D-BF) 
can be realized by the proposed PM-MIMO system, and the generated beams can be steered and varied at $X-Y, X-Z$, and $Y-Z$ planes, respectively. The system architectures of diversity and multiplexing schemes achieved by polarized 3D beams are provided based on the proposed PM-MIMO array system. A robust array selection scheme for $3 \mathrm{D}-\mathrm{BF}$ applications is additionally proposed to efficiently optimize the beam-width and to enhance system performance by the exploration of diversity and multiplexing gains.

Normally in M-MIMO systems, when the number of BS antennae grows large, the size of M-MIMO channel matrix grows large. The vector of M-MIMO channel matrix becomes very long and any two of them are pairwise orthogonal. However, in a space-limited system, pairwise orthogonality cannot be maintained because the adjacent $\mathrm{AE}$ space is usually set equal to or to less than a half signal wavelength. Therefore, for this paper, we additionally modified a conventional blind channel estimation (BCE) approach to exploit the pairwise orthogonality according to the particular characteristics of PM-MIMO systems. That is, the polarized cross-branch links in the system are usually uncorrelated [17]. The proposed BCE approach is presented for PM-MIMO to avoid the pilot contamination and to enhance the system spectrum efficiency. By applying our proposals under the polarized MIMO channel model, Monte Carlo simulations finally confirm the validity of our proposals.

The remaining parts of this paper are organized as follows: Section 2 describes the proposed PM-MIMO array system, and the proposed AE selection scheme is provided in Section 3. In Section 4, a BCE approach for PM-MIMO is introduced, and the simulation results are demonstrated and discussed in Section 5. Finally, our conclusions are drawn in Section 6 .

\section{PM-MIMO Array System}

Figure 1 provides an example of a uniform linear array (ULA) with antenna polarization in the array and branch $(\mathrm{A} \& \mathrm{~B})$ multiple antennae configuration, where three orthogonally colocated antenna branches are fixed at each AE (i.e., antenna port). The beam-width is proved to be relevant to array configuration, where it is inversely proportional to the number of AEs and array element spacing. Because the spacing of three colocated branches at each AE is set to zero, as shown in Figure 1, which makes beam-width scale up to $360^{\circ}$, the beams should be generated via corresponding cross-array branches rather than the colocated branches at each AE [16]. Therefore, we can obtain three orthogonal beams generated by a polarized ULA as follows:

(i) Beam steered in $X-Y$ plane is generated by the branches set of $A_{p} B_{1}$, where $p$ is the index of AE.

(ii) Beam steered in $X-Z$ plane is generated by the branches set of $A_{p} B_{2}$.

(iii) Beam steered in $Y-Z$ plane is generated by the branches set of $A_{p} B_{3}$.

Let $P_{B_{1}}\left(\theta, \phi, \theta_{0,1}\right), \quad P_{B_{2}}\left(\theta, \phi, \theta_{0,2}\right), \quad$ and $\quad P_{B_{3}}\left(\theta, \phi, \theta_{0,3}\right)$ represent the beam radiation patterns generated by the

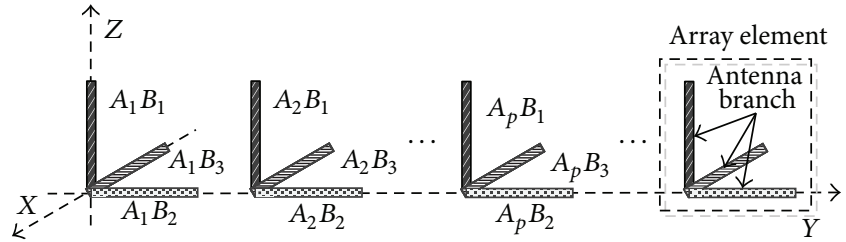

FIGURE 1: Uniform linear array with antenna polarization.

above three sets $A_{p} B_{1}, A_{p} B_{2}$, and $A_{p} B_{3}$, respectively. We have

$$
\begin{aligned}
P_{B_{1}}\left(\theta, \phi, \theta_{0,1}\right) & =\sum_{n=1}^{N} e^{j(n-1)(2 \pi / \varepsilon)\left(\sin \theta-\sin \theta_{0,1}\right)} \\
& =\frac{\sin \left((\pi N / \varepsilon)\left(\sin \theta-\sin \theta_{0,1}\right)\right)}{N \cdot \sin \left((\pi / \varepsilon)\left(\sin \theta-\sin \theta_{0,1}\right)\right)}, \\
P_{B_{2}}\left(\theta, \phi, \theta_{0,2}\right) & =\sum_{n=1}^{N} e^{j(n-1)(2 \pi / \varepsilon)\left(\sin \phi-\sin \theta_{0,2}\right)} \\
& =\frac{\sin \left((\pi N / \varepsilon)\left(\sin \phi-\sin \theta_{0,2}\right)\right)}{N \cdot \sin \left((\pi / \varepsilon)\left(\sin \phi-\sin \theta_{0,2}\right)\right)}, \\
P_{B_{3}}\left(\theta, \phi, \theta_{0,3}\right) & =\sum_{n=1}^{N} e^{j(n-1)(2 \pi / \varepsilon)\left(\sin (\pi / 2-\phi)-\sin \theta_{0,3}\right)} \\
& =\frac{\sin \left((\pi N / \varepsilon)\left(\cos \phi-\sin \theta_{0,3}\right)\right)}{N \cdot \sin \left((\pi / \varepsilon)\left(\cos \phi-\sin \theta_{0,3}\right)\right)},
\end{aligned}
$$

where $\theta$ and $\phi$ denote the azimuth and elevation angle of the radiation pattern; $\varepsilon$ is the array element spacing factor defined by [16], and $\theta_{0,1}, \theta_{0,2}$, and $\theta_{0,3}$ are the off bore-sight angles corresponding to $A_{p} B_{1}, A_{p} B_{2}$, and $A_{p} B_{3}$, respectively [16]. Figure 2(a) with $N=8$ then depicts the 3D beams generated by a triple polarized ULA (TPULA) system via (1), (2), and (3), where three orthogonal beams can be steered and varied separately on the $X-Y, Z-X$, and $Y-Z$ planes. For the second subfigure of Figure $2(\mathrm{a}), \theta_{0,1}, \theta_{0,2}$, and $\theta_{0,3}$ are set as $30^{\circ}$. According to Figure 2(b), a very narrow beam-width is obtained by applying a large number of AEs, for example, $N=$ 64 AEs that performs quite well in terms of BF interferences mitigation.

By employing the TPULA at each multiplexer (MUX), the structure of our proposed PM-MIMO array system is illustrated in Figure 3 with the received signal being given as

$$
y=\sum_{q=1}^{Q} \sum_{b=1}^{3}\left(w_{p, b, q} h_{p, b, q} x_{p, b, q}+n_{p, b, q}\right)
$$

where $h_{p, b, q}$ is the subpolarized channel corresponding to Qth $A_{p} B_{b}$ array element, $w_{p, b, q}$ denotes the $3 \mathrm{D}$-BF weights multiplied at each antenna branch, and $p(1,2, \ldots, P), b$ $(1,2,3)$, and $q(1,2, \ldots, Q)$ represent the $\mathrm{AE}$ index, branch 

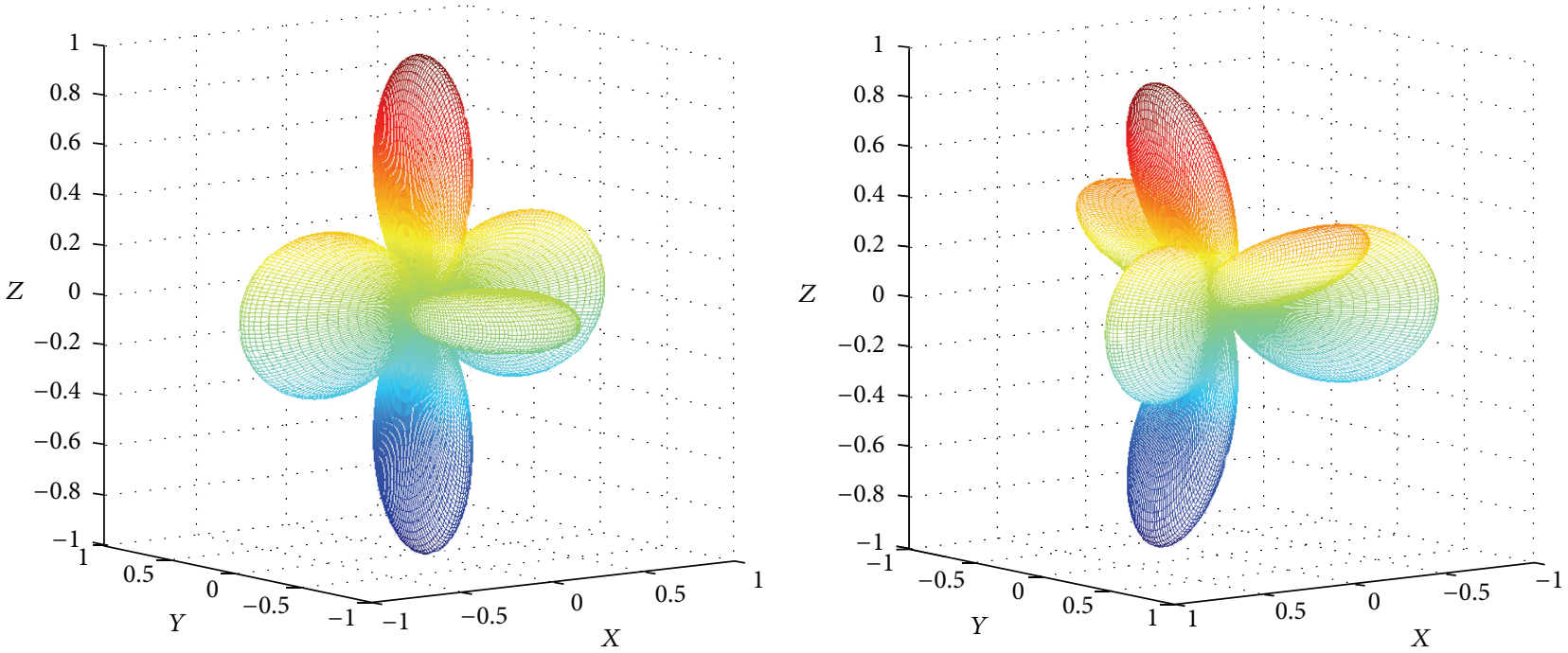

(a) 3D beams generated by a triple-polarized ULA system
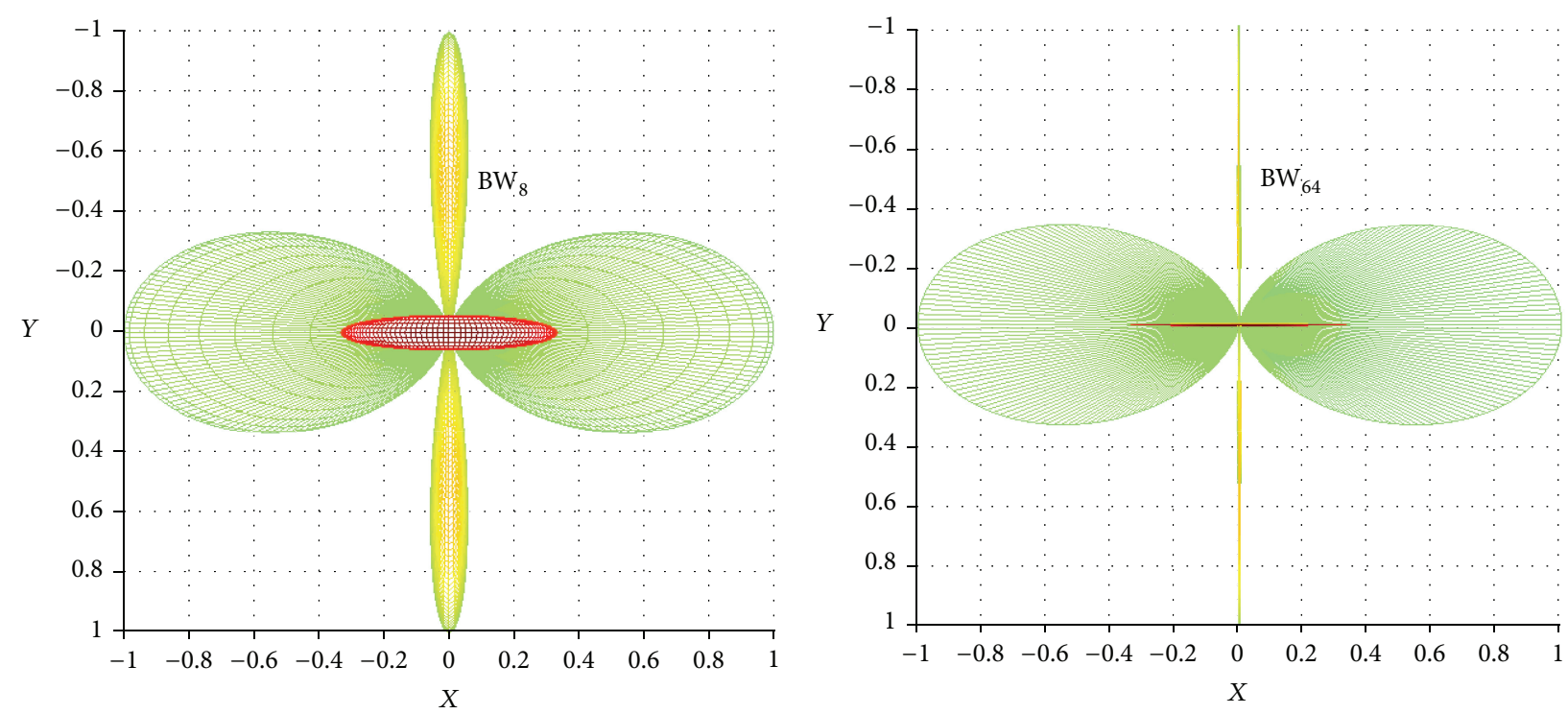

(b) Beam-width varied with different number of AEs being employed, for example, 8 and 64 AEs

FIGURE 2: 3D beams generated by a polarized ULA.

index, and MUX index, respectively. The 3D-BF, which can be applied for multipath reception or combination of relay signals [18], is introduced as a promising technique in M-MIMO systems to enhance the cellular performance by deploying antenna elements in both horizontal and vertical $(\mathrm{H} \& \mathrm{~V})$ dimensions [19]. However, different from the conventional 3D-BF achieved by planar M-MIMO system to control the downtilted beam in a vertical domain, the proposed PMMIMO realizes the $3 \mathrm{D}-\mathrm{BF}$ via the linear combination of polarized beams. It highlights the fact that the $3 \mathrm{D}-\mathrm{BF}$ not only is addressed by the $\mathrm{H} \& \mathrm{~V}$ exploration but also can be achieved by antenna polarization; meanwhile, the antenna polarization also serves as a pivotal solution dedicated to space constraint in M-MIMO systems.

\section{Diversity and Multiplexing Achieved by Polarized 3D Beams with an Array Selection Scheme}

3.1. System Architecture. Figure 4 describes the architecture of the proposed diversity system via polarized 3D beams, where the multiple users with each equipping three colocated antenna branches are considered. As studied by Dao et al. [17], the cross-array links (e.g., from BS $A_{1} B_{1}$ and $A_{2} B_{1}$ to $\mathrm{UE} B_{1}$ ) can be highly correlated by setting the $\mathrm{AE}$ space equal to, or less than, a half-wavelength. The crossbranch links (e.g., from BS $A_{1} B_{1}$ and $A_{1} B_{2}$ to UE $B_{1}$ ), on the other hand, are usually uncorrelated due to the space polarization. This inspired us to incorporate space-time block 


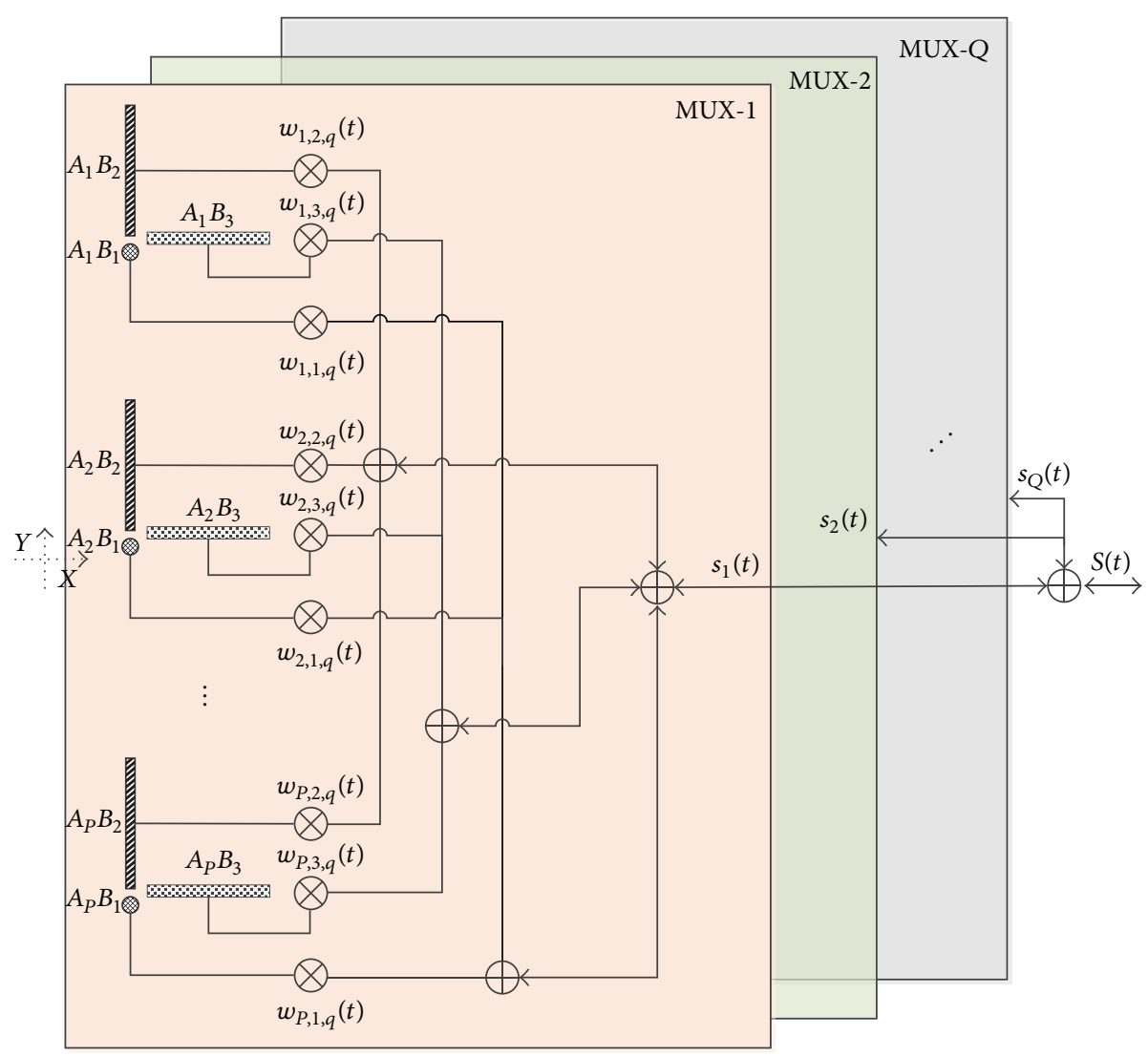

FIGURE 3: Structure of the proposed PM-MIMO array system.

coding (STBC) and BF techniques simultaneously in the TPULA system to boost performance. Moreover, in order to achieve full-rate coding with an odd number of transmit antennae, quasi-orthogonal STBC (QO-STBC) has emerged in the literature $[20,21]$, which fully explores diversity gain but increases the complexity of decoding due to nonorthogonal interference. In this paper, the proposed diversity scheme combines QO-STBC for three transmit antennae and $\mathrm{BF}$ techniques via the TPULA system as illustrated in Figure 4.

In Figure 4 , the BF weights $\left(w_{p_{b}}^{\mathrm{Tx}}\right)$ are multiplied before the inverse fast Fourier transform (IFFT) block of the BS. $\mathbf{X}_{p}$ in (5) is the transmitted QO-STBC symbol matrix [20]:

$$
\mathbf{X}_{p}=\left(\begin{array}{ccc}
x_{\mathrm{II}} & x_{\mathrm{III}} & x_{\mathrm{IV}} \\
x_{\mathrm{I}}^{*} & -x_{\mathrm{IV}}^{*} & x_{\mathrm{III}}^{*} \\
x_{\mathrm{IV}} & x_{\mathrm{I}} & x_{\mathrm{II}} \\
x_{\mathrm{III}}^{*} & -x_{\mathrm{II}}^{*} & x_{\mathrm{I}}^{*}
\end{array}\right)_{I-\text { by-J }},
$$

where the Roman numeral is the symbol index, $i$ is the time index modulo of $4(I=4)$, and $j$ is the antenna index modulo of $3(J=3)$. At each UE, the QO-STBC decoding is applied after the FFT block at each antenna branch [20], and then we can have the received signal for each branch of a specific UE before QO-STBC decoding as

$$
\begin{aligned}
\mathbf{R}_{p \sim i \mathrm{thUE}_{b}} & \left(\mathbf{h}_{p \sim i \mathrm{thUE}_{b}}\left(\mathbf{W}_{p \sim i \mathrm{thUE}_{b}}^{\mathrm{Tx}} \odot \mathbf{X}_{p \sim i \mathrm{thUE}_{b}}\right)^{T}\right)^{T} \\
& +\mathbf{n}_{p \sim i \mathrm{thUE}_{b}} \\
= & \left(\mathbf{W}_{p \sim i \mathrm{thUE}_{b}}^{\mathrm{Tx}} \odot \mathbf{X}_{p \sim i \mathrm{thUE}_{b}}\right) \mathbf{h}_{p \sim i \mathrm{thUE}_{b}}^{T}+\mathbf{n}_{p \sim i \mathrm{thUE}_{b}} \\
= & \left(r_{p \sim i \mathrm{thUE}_{b}, \mathrm{I}} r_{p \sim i \mathrm{thUE}_{b}, \mathrm{II}} r_{p \sim i \mathrm{thUE}_{b}, \mathrm{III}} r_{p \sim i \mathrm{thUE}_{b}, \mathrm{IV}}\right)^{T},
\end{aligned}
$$

where " $\odot$ " denotes the Hadamard product and $\mathbf{W}_{p}^{\mathrm{Tx}}$ is the TxBF weighting matrix given by

$$
\mathbf{W}_{p \sim i \text { thUE }_{b}}^{\mathrm{Tx}}=\left(\begin{array}{ccc}
w_{p_{1}}^{\mathrm{Tx}} & w_{p_{2}}^{\mathrm{Tx}} & w_{p_{3}}^{\mathrm{Tx}} \\
w_{p_{1}}^{\mathrm{Tx}} & w_{p_{2}}^{\mathrm{Tx}} & w_{p_{3}}^{\mathrm{Tx}} \\
w_{p_{1}}^{\mathrm{Tx}} & w_{p_{2}}^{\mathrm{Tx}} & w_{p_{3}}^{\mathrm{Tx}} \\
w_{p_{1}}^{\mathrm{Tx}} & w_{p_{2}}^{\mathrm{Tx}} & w_{p_{3}}^{\mathrm{Tx}}
\end{array}\right) .
$$

$\mathbf{h}_{p \sim i t h U E_{b}}$ is the vector of the polarized cross-branch links, given by

$$
\mathbf{h}_{p \sim i \mathrm{thUE}_{b}}=\left(\begin{array}{lll}
h_{p_{1} \sim i \mathrm{thUE}_{b}} & h_{p_{2} \sim i \mathrm{thUE}_{b}} & h_{p_{3} \sim i \mathrm{thUE}_{b}}
\end{array}\right),
$$




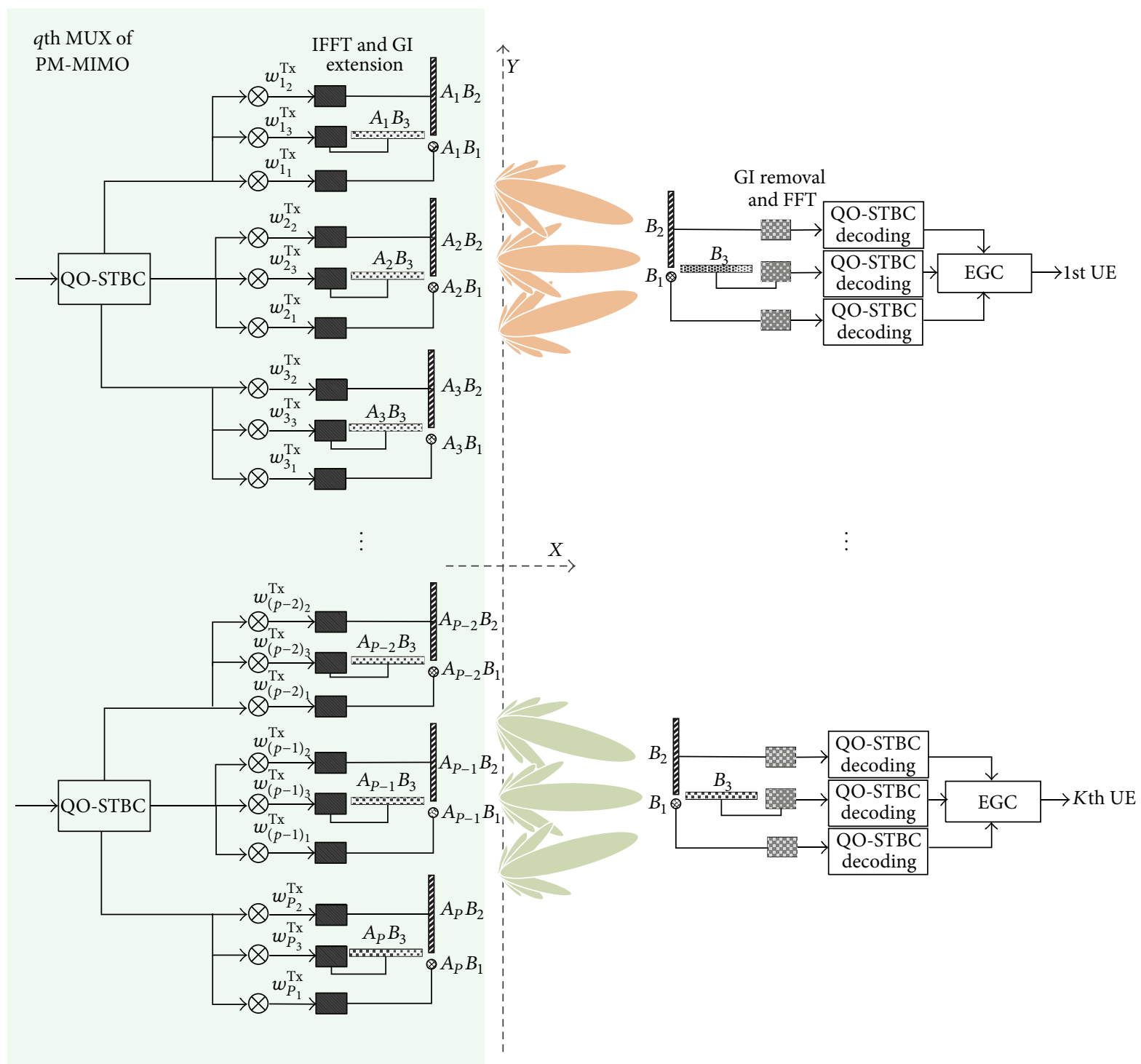

FIGURE 4: Diversity system architecture by polarized 3D beams.

and $\mathbf{n}_{p \sim i \text { thUE }}$ is the noise vector given by

$$
\begin{aligned}
& \mathbf{n}_{p \sim i \mathrm{thUE}_{b}} \\
& =\left(\begin{array}{llll}
\sigma_{p \sim i \mathrm{thUE}_{b}, \mathrm{I}} & \sigma_{p \sim i \mathrm{thUE}_{b}, \mathrm{II}} & \sigma_{p \sim i \mathrm{thUE}}, \mathrm{III} & \sigma_{p \sim i \mathrm{thUE}_{b}, \mathrm{IV}}
\end{array}\right)^{T} .
\end{aligned}
$$

The STBC decoding applied thereafter gives

$$
\begin{aligned}
& \widetilde{\mathbf{X}}_{p \sim i \mathrm{thUE}_{b}}=\mathbf{D}_{p \sim i \mathrm{thUE}_{b}} \mathbf{T}_{p \sim i \mathrm{thUE}_{b}} \\
& =\left(\begin{array}{llll}
\tilde{x}_{p \sim i \mathrm{thUE}_{b}, \mathrm{I}} & \tilde{x}_{p \sim i \mathrm{thUE}_{b}, \mathrm{II}} & \tilde{x}_{p \sim i \mathrm{thUE}_{b}, \mathrm{III}} & \tilde{x}_{p \sim i \mathrm{thE}_{b}, \mathrm{IV}}
\end{array}\right)^{T} .
\end{aligned}
$$

Here, $\mathbf{D}_{p \sim q_{b}}$ is the STBC decoding matrix, which is modified based on equation (12) in [20] by considering TxBF weights:

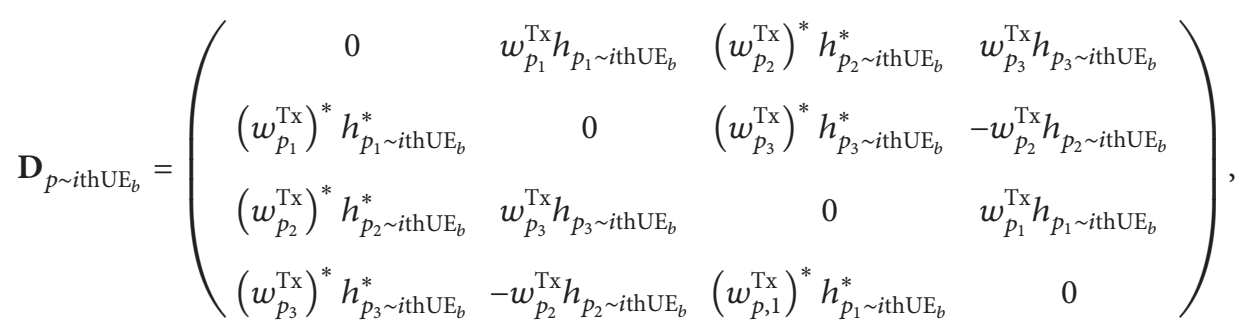


where $\mathbf{T}_{p \sim q_{b}}$ is defined as

$$
\begin{aligned}
& \mathbf{T}_{p \sim i \mathrm{thUE}_{b}} \\
& \quad=\left(\begin{array}{llll}
\widetilde{r}_{p \sim i \mathrm{thUE}}, \mathrm{I} & \widetilde{r}_{p \sim i \mathrm{thUE}_{b}, \mathrm{II}}^{*} & \widetilde{r}_{p \sim i \mathrm{thUE}}, \mathrm{III} & \widetilde{r}_{p \sim i t h U E_{b}, \mathrm{IV}}^{*}
\end{array}\right)^{T}
\end{aligned}
$$

by taking the conjugate of the second and the fourth elements of $\mathbf{R}_{p \sim i t h U E_{b}}$. The final output is obtained by the functional block of equal gain combining (EGC) as

$\widetilde{\mathbf{X}}$

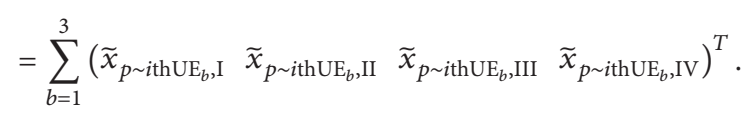

Figure 5 describes the architecture of multiplexing system by polarized $3 \mathrm{D}$ beams, where each generated beam is dedicated to a piece of UE. In this case, the data rate is three times higher than the 3D-BF diversity system. Here, we also consider multiple users, with each of them equipping three colocated antenna branches, and note that, in these two proposed schemes, the zero-forcing BF is assumed and applied at transmitter $\mathrm{Tx}$ (i.e., the BS). Compared with diversity case, the proposed multiplexing scheme is relatively simple that the received signal for a specific UE after EGC process can be given as

$$
\begin{aligned}
& \mathbf{R}_{p \sim i \mathrm{thUE}_{b}} \\
& =\sum_{b=1}^{3}\left(\mathbf{h}_{p \sim i \mathrm{thUE}_{b}}\left(\mathbf{W}_{p \sim i \mathrm{thUE}_{b}}^{\mathrm{Tx}} \odot \mathbf{S}_{p \sim i \mathrm{thUE}_{b}}\right)+\mathbf{n}_{p \sim i \mathrm{thUE}_{b}}\right) .
\end{aligned}
$$

3.2. Array Selection Scheme for PM-MIMO Array System. The sharp beam as illustrated in Figure 2(b) performs quite well in terms of $\mathrm{BF}$ interferences mitigation. However, it is not efficient to assign all AEs of a MUX to form one beam, especially for cell-edge UEs. Overall, AEs in a MUX can be divided into several groups to form beams for separate UEs, and this part analyses the minimum number of AEs required to mitigate $\mathrm{BF}$ interferences.

Let us have two simultaneous beams point two adjacent pieces of UEs, as demonstrated in Figure 6, where $L$ gives the beam coverage and $D$ and $d$ denote the distance from UE to BS and the distance between the adjacent UEs, respectively. To make the analysis meaningful, we assume that there is a sufficient amount of UE deployed in a cell, and therewith, the UEs are close each other, resulting in $d_{1} \approx v_{1}$ and $d_{2} \approx v_{2}$. According to Figure 6, in the case of UE (UE-1 and UE-2) located at a $3 \mathrm{~dB}$ beam area, that is, a half power beam-width (HPBW) area, the HPBW needs to be controlled at less than $2 d_{1}$ to avoid $\mathrm{BF}$ interferences. On the other hand, when UEs (UE-3 and UE-4) are located at a beam peak area, the HPBW can be set larger than $2 d_{2}$. In addition to assuming that UE is distributed in a square cell, which can be treated as a city block for horizontal BF or a building for vertical BF, we provide Figure 7 for calculating average $D$ and $d$. Suppose that UEs follow a spatial Poisson process with an intensity of $\rho$, so the number of UEs in a cell is given as

$$
K=\rho L^{2} .
$$

The average distance between the BS and UE is calculated by

$$
\begin{aligned}
D & =\iint_{L^{2}} \frac{1}{L^{2}} r d r d \theta \\
& =\frac{2}{L^{2}}\left(\int_{0}^{\omega} \int_{0}^{a / \cos \theta} r^{2} d r d \theta+\int_{\omega}^{\pi / 2} \int_{0}^{2 a / \sin \theta} r^{2} d r d \theta\right) \\
& =\frac{\xi L}{2}
\end{aligned}
$$

where $\xi=\ln (2+\sqrt{5}) / 12+\sqrt{5}-2 \ln ((\sqrt{5}-1) / 2) / 3 \approx 1.187$ The average distance between two adjacent pieces of UEs is given as

$$
d=\iint_{A} \frac{1}{A} r d r d \theta=\int_{0}^{2 \pi} \int_{0}^{r_{\mathrm{UE}}} \frac{r^{2}}{\pi r_{\mathrm{UE}}^{2}} d r d \theta=\frac{2}{3} r_{\mathrm{UE}}
$$

where $A$ denotes the local coverage area of UE with a radius of $r_{\mathrm{UE}}$ depending on $\rho$.

Because $D$ is calculated close to $L / 2$, the HPBW $<2 d$ needs to be maintained to avoid BF interferences. According to Su and Chang [16], we have

$$
\mathrm{HPBW} \approx 2\left|\arcsin \left(1.391 \frac{\varepsilon}{\pi P}+\sin \theta_{0}\right)\right|<\frac{4}{3} r_{\mathrm{UE}},
$$

where $\varepsilon$ denotes the array spacing factor; that is, $\varepsilon=\lambda / s$ with $s$ and $\lambda$ representing the array spacing and signal wavelength, respectively. $\theta_{0}$ is the signal incidence angle shifted from the bore-sight direction, and the antenna bore-sight is the axis vertical to the orientation of the array alignment. For example, if the radius of a user's local area $\left(r_{\mathrm{UE}}\right)$ is 15 meters, at least $8 \mathrm{AEs}$ are required to avoid BF interferences when $\varepsilon=2$ and $\theta_{0}=\pi / 4$. Please note that the above analysis is derived based on the isotropic array antenna system. The required number of AEs may decrease by using the dipole antenna because it does not radiate in the longitudinal direction of an antenna structure that maintains a higher radiation gain compared with the isotropic antenna.

As discussed in Su and Chang [16] and Liu [22], the beamwidth is increased significantly when the beam steers to an angle far off the bore-sight direction, such as the case of $\theta_{0}$ in (18) reaching $90^{\circ}$. In order to avoid beam-with extension, we propose a scheme to cope with the large off bore-sight angle by dynamically selecting the set of polarized branches for 3D$\mathrm{BF}$ that can effectively work without increasing the dimension of the array system. As depicted in Figure 8, let $\alpha_{k}, \beta_{k}$, and $\gamma_{k}$ denote the acute angles corresponding to the $X, Y$, and $Z$ axes of the $k$ th incident signal; we have

$$
\begin{aligned}
& \alpha_{k}=\arcsin \frac{\sqrt{b_{k}^{2}+c_{k}^{2}}}{D_{k}}, \\
& \beta_{k}=\arcsin \frac{\sqrt{a_{k}^{2}+c_{k}^{2}}}{D_{k}}, \\
& \gamma_{k}=\arcsin \frac{\sqrt{a_{k}^{2}+b_{k}^{2}}}{D_{k}},
\end{aligned}
$$



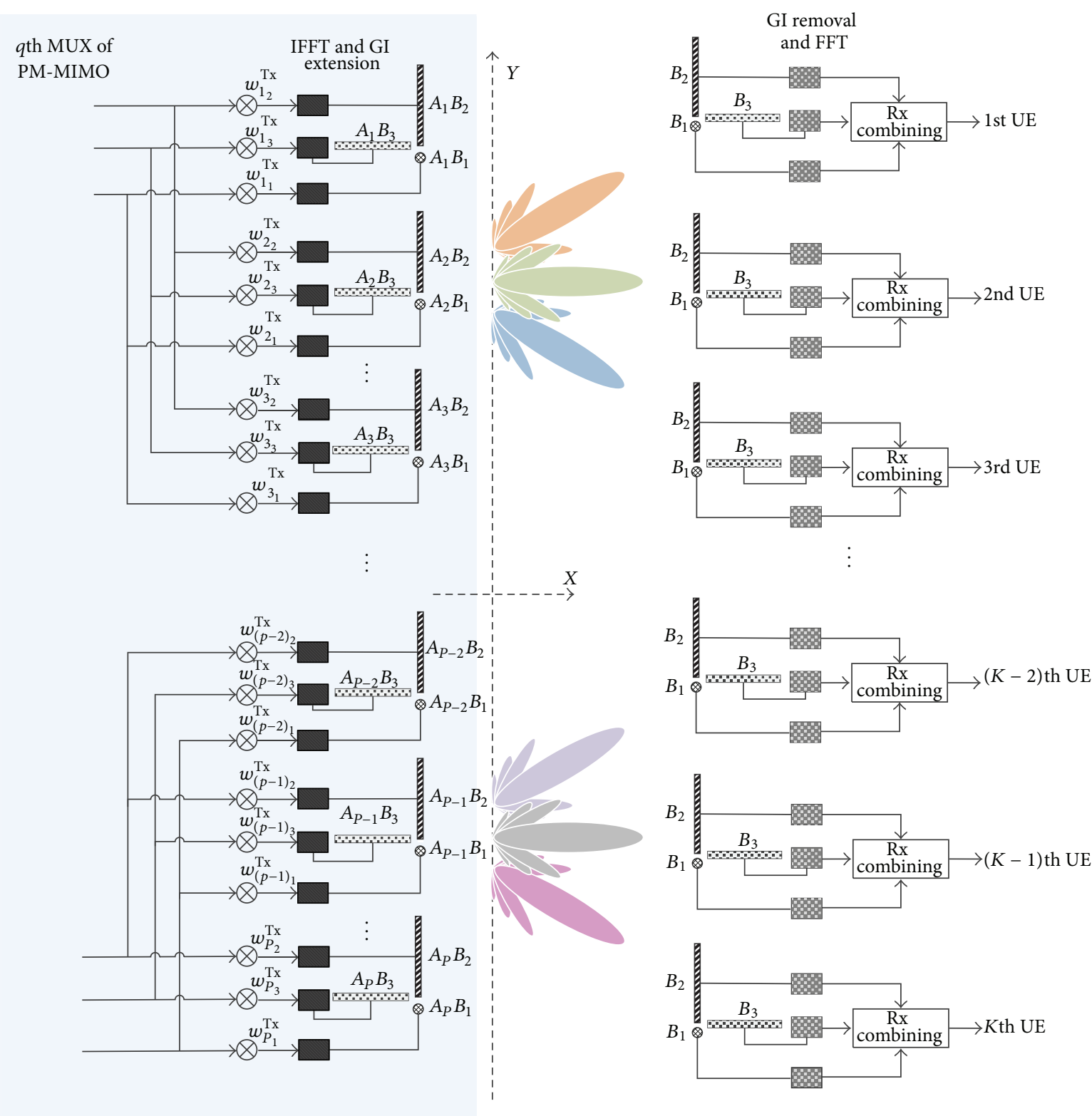

Figure 5: Multiplexing system architecture by polarized 3D beams.

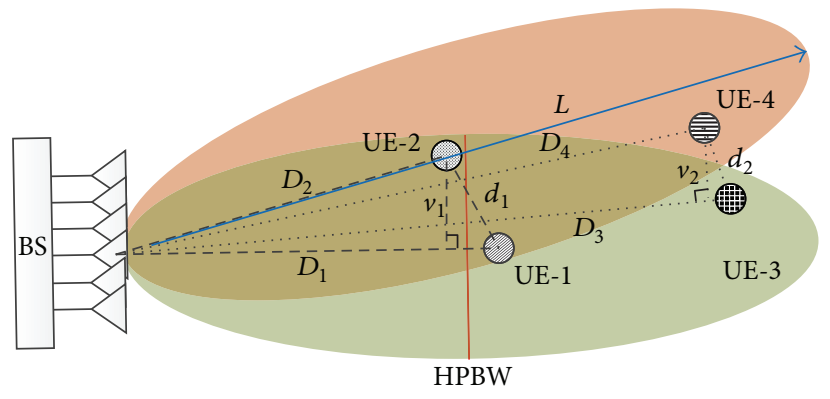

Figure 6: Two beams steer toward two adjacent pieces of UE. 


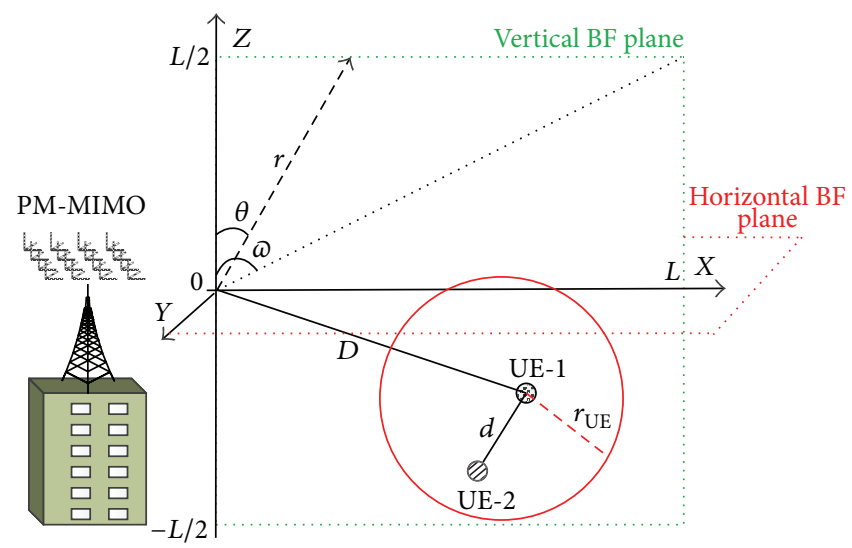

Figure 7: Illustration of a square cell used for calculating $D$ and $d$.

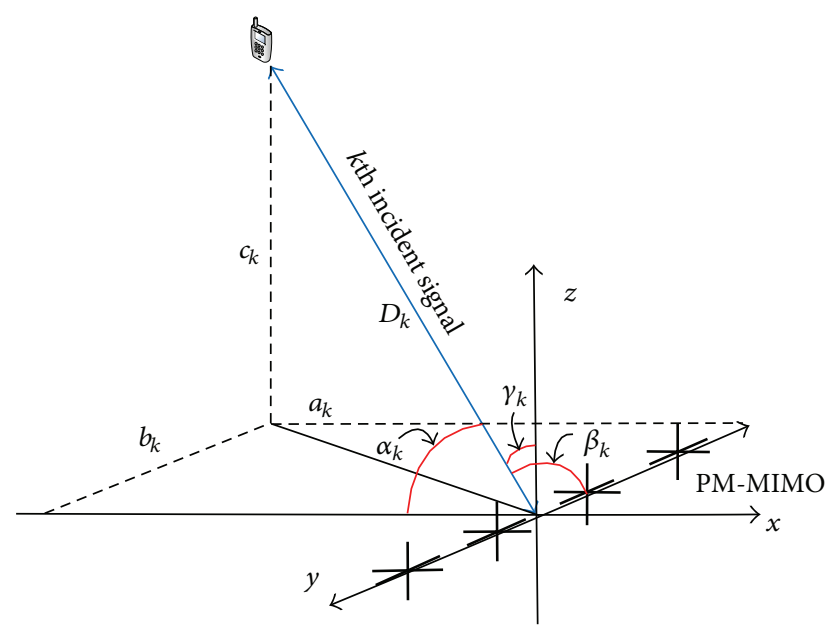

FigURE 8: Selection on the set of branches for 3D-BF via incident signal.

where $\alpha_{k}, \beta_{k}$, and $\gamma_{k} \in(0, \pi / 2)$. In order to avoid beamwidth extension by considering the off bore-sight angle, the following criteria need to be carried out for $3 \mathrm{D}-\mathrm{BF}$ applications:

(i) When $\alpha_{k}=\max \left(\alpha_{k}, \beta_{k}, \gamma_{k}\right)$, use the set of branches of $A_{p} B_{3}$ to form the beam for the $k$ th incident signal.

(ii) When $\beta_{k}=\max \left(\alpha_{k}, \beta_{k}, \gamma_{k}\right)$, use the set of branches of $A_{p} B_{2}$ to form the beam for the $k$ th incident signal.

(iii) When $\gamma_{k}=\max \left(\alpha_{k}, \beta_{k}, \gamma_{k}\right)$, use the set of branches of $A_{p} B_{1}$ to form the beam for the $k$ th incident signal.

Let $y_{1}(t), y_{2}(t), \ldots, y_{k}(t)$ denote the incident signal sequences that come from random directions; Figure 9 then provides the flowchart of the proposed AE selection scheme for the PM-MIMO system. According to Figure 9, the proposed scheme at first detects the incident signal sequences and determines the minimum number of AEs used for 3D$\mathrm{BF}$ via the criterion provided by (18). And then, the proposed scheme categorizes the incident signal sequences into three categories according to $\max \left(\alpha_{k}, \beta_{k}, \gamma_{k}\right)$. For each category, the proposed schemes prepare the set of branches used for 3D$\mathrm{BF}$ via the criteria provided before. If the $3 \mathrm{D}-\mathrm{BF}$ application is dedicated to the cell-edge users, the diversity by using polarized 3D beams is employed to maintain the cell-edge users' performances. Otherwise, multiplexing via polarized $3 \mathrm{D}$ beams is suggested in order to increase overall system throughput.

\section{Blind Channel Estimation to Avoid Pilot Contamination in PM-MIMO Array System}

Theoretically, the M-MIMO system is proved to have many attractive features in wireless communications. However, these features are obtained mainly based on the perfect channel estimation. Practically, the BS does not have perfect channel state information that limits the exploration of $\mathrm{M}$ MIMO systems. Moreover, conventional channel estimation by using training sequences may not be applicable to $\mathrm{M}$ MIMO systems because usually there are tens or hundreds of antennae applied in M-MIMO systems. Spectrum efficiency could not only be decreased dramatically by reserving many pilots for channel estimation, but pilot contamination also limits performance because the pilot positions in a resource block have to be reused due to massive antenna employment [23-26]. 


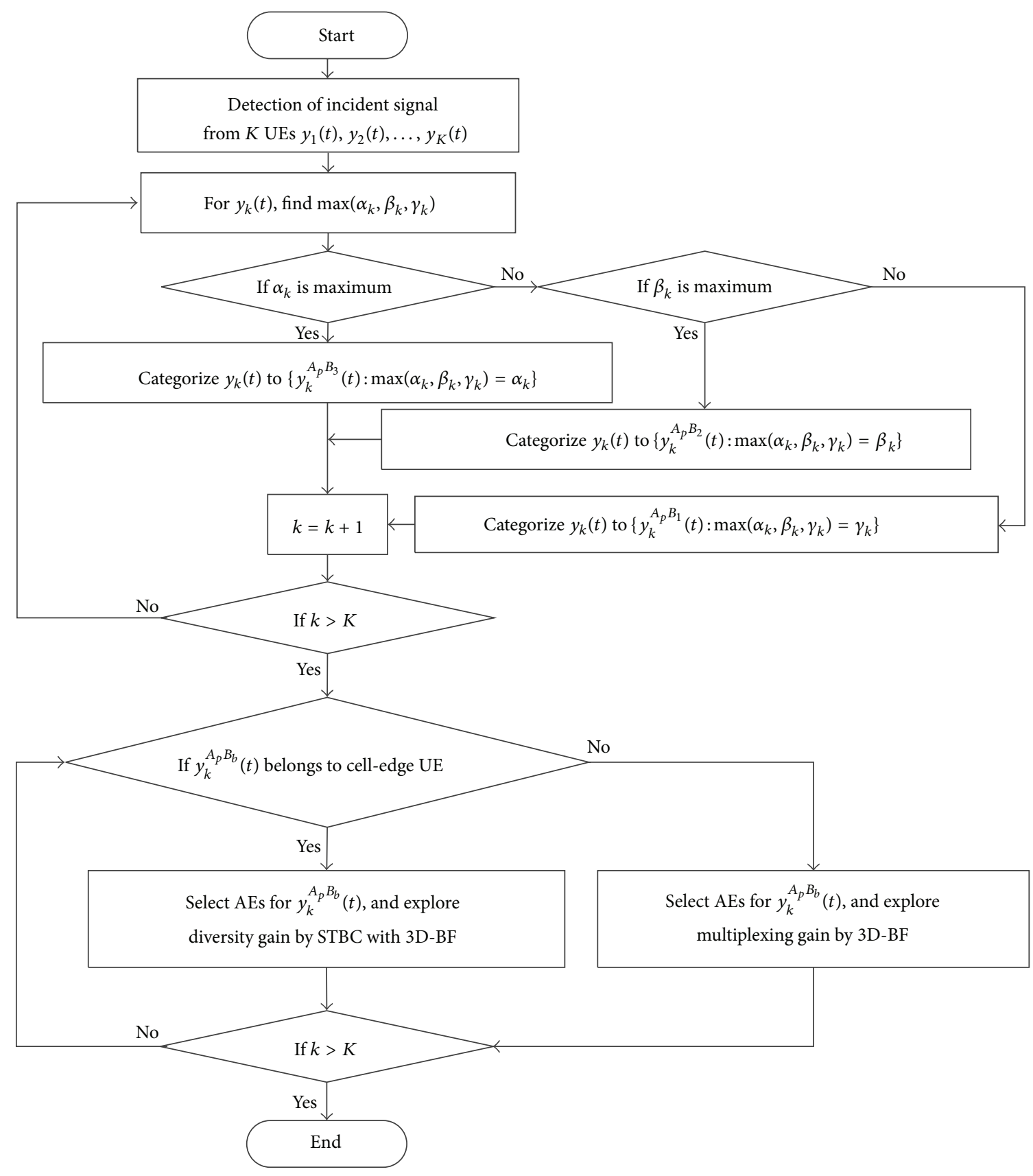

FIgURE 9: Flowchart of the proposed AE selection scheme for PM-MIMO system.

The BCE approach, which requires no, or a minimal number of, pilots needs to be applied in M-MIMO systems. One of the BCE strategies is based on eigenvalue decomposition (EVD) for the covariance matrix of a received signal that needs to preserve pairwise orthogonality among channel vectors [27-29]. Let us define an M-MIMO system model as

$$
\mathbf{y}_{k}=\mathbf{H} \mathbf{x}_{k}+\mathbf{n}_{k}
$$

where $\mathbf{x}_{k}$ is the transmitted symbols, $\mathbf{H}$ is an $M$-by- $N$ channel matrix between the BS and the $k$ th $\mathrm{UE}$, and $\mathbf{n}_{k}$ denotes the additive white noise. The covariance matrix of the received signal is then defined by

$$
\mathbf{R}_{\mathbf{y}_{k}} \triangleq \mathbf{y}_{k} \mathbf{y}_{k}^{H}=\mathbf{H R}_{\mathbf{x}_{k}} \mathbf{H}^{H}+\mathbf{R}_{\mathbf{n}_{k}},
$$

where $H$ represents the Hermitian Transpose operation. Additionally multiplying $\mathbf{H}$ at both sides of (21), we have

$$
\mathbf{R}_{\mathbf{y}_{k}} \mathbf{H}=\mathbf{H R}_{\mathbf{x}_{k}} \mathbf{H}^{H} \mathbf{H}+\mathbf{H} .
$$


From the law of large number, the channel vectors between the BS and the deployed UEs become very long, random, and pairwise orthogonal, which satisfies the condition

$$
\frac{1}{M} \mathbf{H}^{H} \mathbf{H} \longrightarrow \mathbf{I}_{N}, \quad \text { as } M \longrightarrow \infty \text {. }
$$

Thereafter, the estimated channel can be obtained as the eigenvectors of $\mathbf{R}_{\mathbf{y}_{k}}$ via EVD processing [29].

Note that since we focus on the link-level performance, the element of channel matrix $\mathbf{H}$ should be normalized, and only the additive noise and fast fading effects are taken into consideration to generate $\mathbf{H}$. If the array spacing is larger than a half wave-length, the elements of $\mathbf{H}$ would be lowly correlated, and then the condition of (23) is easy to be satisfied as reported elsewhere [30], where the BS AEs are usually separated by several wave-lengths resulting in an uncorrelated Tx radiation pattern to preserve the pairwise orthogonality among channel vectors. However, in space-limited M-MIMO systems, the pairwise orthogonality cannot be maintained because the adjacent $\mathrm{AE}$ space is fixed, normally, at equal

$$
\mathbf{h}_{\mathrm{Tx}_{p}-\mathrm{Rx}_{\hat{p}}}(t)=\sqrt{\frac{\eta}{I}} \sum_{i=1}^{I}\left(\begin{array}{c}
h_{\mathrm{Tx} A_{p} B_{1}-\mathrm{Rx} A_{\hat{p}} B_{1}}(t) \\
\sqrt{\mu} \cdot h_{\mathrm{Tx} A_{p} B_{1}-\mathrm{Rx} A_{\hat{p}} B_{2}}(t) \\
\sqrt{\mu} \cdot h_{\mathrm{Tx} A_{p} B_{1}-\mathrm{Rx}_{\hat{p}} B_{3}}(t)
\end{array}\right.
$$

Here, $\eta$ and $I$ denote the transmit power and the number of scatterers, respectively. Channel matrix (24) has a size of $3 P$-by- $3 \widehat{P}$, which is composed of 3 -by- 3 submatrices. Each submatrix holds the polarized cross-branch links between the $P$ th AE of Tx (the BS) and the $\widehat{P}$ th AE (UE) of receiver (Rx). Please note that throughout the paper the UE is assumed with one $\mathrm{AE}$; that is, $\widehat{P}=1$. The channel vectors of submatrix (25) are random and pairwise orthogonal because the polarized links from a Tx AE with three orthogonal branches to a single Rx branch are usually highly orthogonal [17]. Consequently, we can apply the EVD-based BCE via submatrix of (25). By redefining the covariance matrix of the received signal as

$$
\mathbf{R}_{\mathbf{y}_{k}} \triangleq \mathbf{y}_{k} \mathbf{y}_{k}^{H}=\mathbf{h}_{\mathrm{Tx} A_{p}-\mathrm{Rx} A_{\widehat{q}}} \mathbf{R}_{\mathbf{x}_{k}} \mathbf{h}_{\mathrm{Tx} A_{p}-\mathrm{Rx} A_{\hat{q}}}^{H}+\mathbf{R}_{\mathbf{n}_{k}}
$$

the estimated channel can be obtained as the eigenvectors of $\mathbf{R}_{\mathbf{y}_{k}}$ via EVD processing because the condition of $(1 / 3) \mathbf{h}_{\mathrm{Tx} A_{p}-\operatorname{Rx} A_{\hat{q}}}^{H} \mathbf{h}_{\mathrm{Tx} A_{p}-\mathrm{Rx} A_{\hat{q}}} \rightarrow \mathbf{I}_{3}$ is satisfied.

\section{Performance Verification}

Table 1 lists the parameters settings of the performance verification based on the LTE-A specification [32]. The downlink data mapping for LTE-A resource blocks used in simulations is demonstrated in Figure 10, which is based on the QO-STBC or less than a half signal wave-length. Consequently, in this paper, we modify the EVD-based blind channel estimation scheme studied in Ngo and Larsson [29]. The modified $\mathrm{BCE}$ approach can exploit the pairwise orthogonality via the particular characteristics of PM-MIMO systems; that is, the polarized cross-branch links in the system usually are uncorrelated, even though the adjacent $\mathrm{AE}$ spacing is set equal to, or less than, a half signal wave-length [17].

Based on other researches $[14,16,31]$, an extension of the channel matrix for PM-MIMO systems can be represented as

$$
\begin{aligned}
& \mathbf{H}_{\mathrm{PM}-\mathrm{MIMO}}(t) \\
& \quad=\left(\begin{array}{ccc}
\mathbf{h}_{\mathrm{Tx} A_{1}-\mathrm{Rx} A_{1}}(t) & \cdots & \mathbf{h}_{\mathrm{Tx} A_{1}-\mathrm{Rx} A_{\hat{P}}}(t) \\
\vdots & \ddots & \vdots \\
\mathbf{h}_{\mathrm{Tx} A_{P}-\mathrm{Rx} A_{1}}(t) & \cdots & \mathbf{h}_{\mathrm{Tx} A_{P}-\mathrm{Rx} A_{\hat{P}}}(t)
\end{array}\right),
\end{aligned}
$$

where symbol matrix of (5). We use the training sequences provided by [32], which are defined as

$$
\begin{aligned}
r_{l, n_{s}}(m)= & \frac{1}{\sqrt{2}}(1-2 \cdot c(2 m)) \\
& +j \frac{1}{\sqrt{2}}(1-2 \cdot c(2 m+1)),
\end{aligned}
$$

where $m=0,1, \ldots, 2 N_{R B}^{\max , D L}-1$. The pilot density is $14.3 \%$, and the initialization of $c$ is defined as

$$
\begin{aligned}
c_{\text {init }}= & 2^{10} \cdot\left(7 \cdot\left(n_{s}+1\right)+l+1\right) \cdot\left(2 \cdot N_{\mathrm{ID}}^{\text {cell }}+1\right)+2 \\
& \cdot N_{\mathrm{ID}}^{\text {cell }}+N_{\mathrm{CP}},
\end{aligned}
$$

where $n_{s}$ is the slot number within a frame, and

$$
N_{\mathrm{CP}}= \begin{cases}1 & \text { for normal CP } \\ 0 & \text { for extended CP. }\end{cases}
$$

Figure 11 depicts the probability densities of the HPBW generated in simulations, where the HPBW can be effectively kept as $50^{\circ}$ on average by using the proposed AE selection scheme of Figure 9. However, the average HPBW extends by about $15^{\circ}$ when not considering the off bore-sight angle effect. This demonstrates that the proposed AE selection scheme is robust that can optimize the generated beamwidth to avoid BF interference. 
Branch $A_{1} B_{1}$

\begin{tabular}{|c|c|c|c|c|c|c|c|c|c|c|c|c|c|}
\hline 0 & $x_{2}$ & $x_{1}^{*}$ & $x_{4}$ & $P_{A_{1} B_{1}}$ & $x_{3}^{*}$ & $x_{50}$ & $x_{49}^{*}$ & $x_{52}$ & \begin{tabular}{|l|}
$x_{51}^{*}$ \\
\end{tabular} & $x_{98}$ & $P_{A_{1} B_{1}}$ & \begin{tabular}{|l|l|}
$x_{97}^{*}$ & $x_{100}$ \\
\end{tabular} & $x_{99}^{*}$ \\
\hline 1 & $x_{6}$ & $x_{5}^{*}$ & $x_{8}$ & & $x_{7}^{*}$ & $x_{54}$ & $x_{53}^{*}$ & $x_{56}$ & $x_{55}^{*}$ & $x_{102}$ & & \begin{tabular}{|l|l|}
$x_{101}^{*}$ & $x_{104}$
\end{tabular} & $x_{103}^{*}$ \\
\hline 2 & $x_{10}$ & $x_{9}^{*}$ & $x_{12}$ & & $x_{11}^{*}$ & $x_{58}$ & $x_{57}^{*}$ & $x_{60}$ & $x_{59}^{*}$ & $x_{106}$ & & \begin{tabular}{l|l}
$x_{105}^{*}$ & $x_{108}$
\end{tabular} & $x_{107}^{*}$ \\
\hline 3 & $x_{14}$ & $x_{13}^{*}$ & $x_{16}$ & & $x_{15}^{*}$ & $x_{62}$ & $x_{61}^{*}$ & $x_{64}$ & $x_{63}^{*}$ & $x_{110}$ & & $x_{109}^{*} x_{112}$ & $x_{111}^{*}$ \\
\hline & $x_{18}$ & $x_{17}^{*}$ & $x_{20}$ & & $x_{19}^{*}$ & $x_{66}$ & $x_{65}^{*}$ & $x_{68}$ & $x_{67}^{*}$ & $x_{114}$ & & \begin{tabular}{|l|l}
$x_{113}^{*}$ & $x_{116}$
\end{tabular} & $x_{115}^{*}$ \\
\hline 5 & $x_{22}$ & $x_{21}^{*}$ & $x_{24}$ & & $x_{23}^{*}$ & $x_{70}$ & $x_{69}^{*}$ & $x_{72}$ & $x_{71}^{*}$ & $x_{118}$ & & \begin{tabular}{|l|l|}
$x_{117}^{*}$ & $x_{120}$ \\
\end{tabular} & $x_{119}^{*}$ \\
\hline 6 & $x_{26}$ & $x_{25}^{*}$ & $x_{28}$ & $P_{A_{1} B_{1}}$ & $x_{27}^{*}$ & $x_{74}$ & $x_{73}^{*}$ & $x_{76}$ & $x_{75}^{*}$ & $x_{122}$ & $P_{A_{1} B_{1}}$ & \begin{tabular}{|l|l|l}
$x_{121}^{*}$ & $x_{124}$
\end{tabular} & $x_{123}^{*}$ \\
\hline 7 & $x_{30}$ & $x_{29}^{*}$ & $x_{32}$ & & $x_{31}^{*}$ & $x_{78}$ & $x_{77}^{*}$ & $x_{80}$ & $x_{79}^{*}$ & $x_{126}$ & & $x_{125}^{*} x_{128}$ & $x_{127}^{*}$ \\
\hline 8 & $x_{34}$ & $x_{33}^{*}$ & $x_{36}$ & & $x_{35}^{*}$ & $x_{82}$ & $x_{81}^{*}$ & $x_{84}$ & $x_{83}^{*}$ & $x_{130}$ & & \begin{tabular}{|l|l}
$x_{129}^{*}$ & $x_{132}$ \\
\end{tabular} & $x_{131}^{*}$ \\
\hline 9 & $x_{38}$ & $x_{37}^{*}$ & $x_{40}$ & & $x_{39}^{*}$ & $x_{86}$ & $x_{85}^{*}$ & $x_{88}$ & $x_{87}^{*}$ & $x_{134}$ & & $\begin{array}{lll}x_{133}^{*} & x_{136} \\
\end{array}$ & $x_{135}^{*}$ \\
\hline 10 & $x_{42}$ & $x_{41}^{*}$ & $x_{44}$ & & $x_{43}^{*}$ & $x_{90}$ & $x_{89}^{*}$ & $x_{92}$ & $x_{91}^{*}$ & $x_{138}$ & & \begin{tabular}{|l|l}
$x_{137}^{*}$ & $x_{140}$ \\
\end{tabular} & $x_{139}^{*}$ \\
\hline 11 & $x_{46}$ & $x_{45}^{*}$ & $x_{48}$ & & $x_{47}^{*}$ & $x_{94}$ & $x_{93}^{*}$ & $x_{96}$ & $x_{95}^{*}$ & $x_{142}$ & & \begin{tabular}{|l|l|}
$x_{141}^{*}$ & $x_{144}$ \\
\end{tabular} & $x_{143}^{*}$ \\
\hline$\downarrow$ & 0 & 1 & 2 & 3 & 4 & 5 & 6 & 7 & 8 & 9 & 10 & $\begin{array}{ll}11 \quad 12 \\
\end{array}$ & 13 \\
\hline
\end{tabular}

Branch $A_{2} B_{1}$

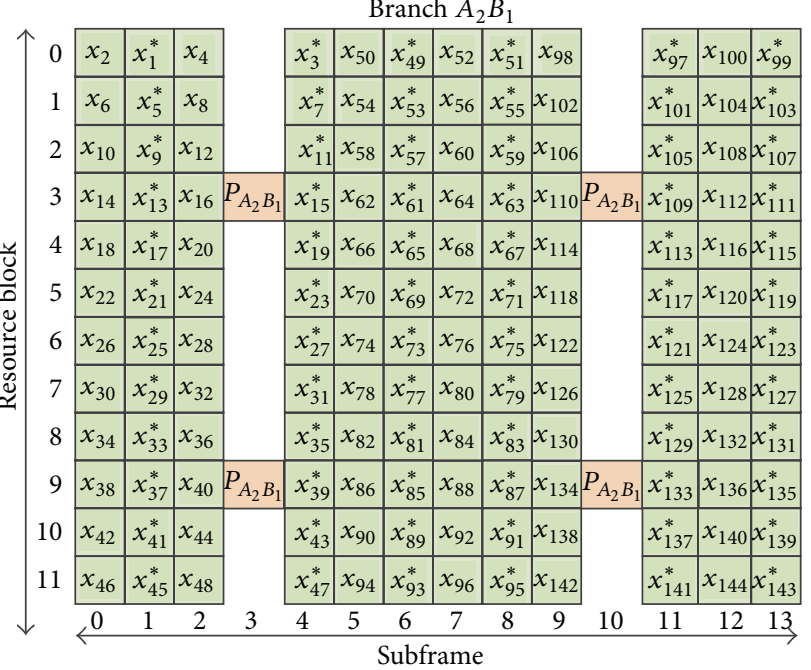

(a) Data mapping at $\operatorname{Tx} A_{p} B_{1}$

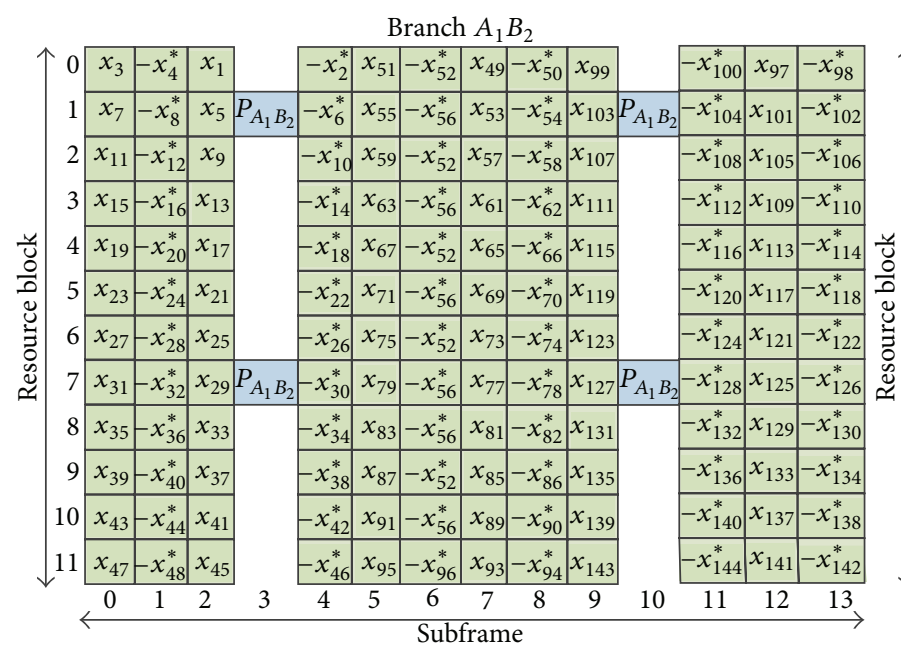

(b) Data mapping at $\operatorname{Tx} A_{p} B_{2}$
Branch $A_{2} B_{2}$

\begin{tabular}{|c|c|c|c|c|c|c|c|c|c|c|c|c|c|}
\hline \multirow{2}{*}{\multicolumn{5}{|c|}{\begin{tabular}{|l|l|l|}
$x_{3}$ & $-x_{1}^{*}$
\end{tabular}}} & & \multirow{2}{*}{\multicolumn{2}{|c|}{$-x_{100}^{*}$}} & \multirow[b]{2}{*}{$x_{97}$} & \multirow{2}{*}{$-x_{00}^{*}$} \\
\hline & & & & & $-x_{2}^{*}$ & $x_{51}$ & $\mid-x_{52}^{*}$ & $x_{49}$ & \begin{tabular}{|l|l|}
$-x_{50}^{*}$ & $x_{99}$ \\
\end{tabular} & & & & \\
\hline & $x_{7}$ & $-x_{8}^{*}$ & $x_{5}$ & & $-x_{6}^{*}$ & $x_{55}$ & $-x_{56}^{*}$ & $x_{53}$ & \begin{tabular}{|l|l|}
$-x_{54}^{*}$ & $x_{103}$ \\
\end{tabular} & & $-x_{104}^{*}$ & $x_{101}$ & $-x_{102}^{*}$ \\
\hline & $x_{11}$ & $-x_{12}^{*}$ & $x_{9}$ & & $-x_{10}^{*}$ & $x_{59}$ & $-x_{52}^{*}$ & $x_{57}$ & \begin{tabular}{|l|l|}
$-x_{58}^{*}$ & $x_{107}$ \\
\end{tabular} & & $-x_{108}^{*}$ & $x_{105}$ & $-x_{106}^{*}$ \\
\hline & $x_{15}$ & $-x_{16}^{*}$ & $x_{13}$ & & $-x_{14}^{*}$ & $x_{63}$ & $-x_{56}^{*}$ & $x_{61}$ & \begin{tabular}{|l|l|}
$-x_{62}^{*}$ & $x_{111}$ \\
\end{tabular} & & $-x_{112}^{*}$ & $x_{109}$ & $-x_{110}^{*}$ \\
\hline & $x_{19}$ & $-x_{20}^{*}$ & $x_{17}$ & $P_{A_{2} B_{2}}$ & $-x_{18}^{*}$ & $x_{67}$ & $-x_{52}^{*}$ & $x_{65}$ & \begin{tabular}{|l|l|}
$-x_{66}^{*}$ & $x_{115}$ \\
\end{tabular} & $P_{A_{2} B}$ & $-x_{116}^{*}$ & $x_{113}$ & $-x_{114}^{*}$ \\
\hline & $x_{23}$ & $-x_{24}^{*}$ & $x_{21}$ & & $-x_{22}^{*}$ & $x_{71}$ & $-x_{56}^{*}$ & $x_{69}$ & \begin{tabular}{|l|l|}
$-x_{70}^{*}$ & $x_{119}$ \\
\end{tabular} & & $-x_{120}^{*}$ & $x_{117}$ & $-x_{118}^{*}$ \\
\hline & $x_{27}$ & $-x_{28}^{*}$ & $x_{25}$ & & $-x_{26}^{*}$ & $x_{75}$ & $-x_{52}^{*}$ & $x_{73}$ & \begin{tabular}{|l|l|}
$-x_{74}^{*}$ & $x_{123}$ \\
\end{tabular} & & $-x_{124}^{*}$ & $x_{121}$ & $-x_{122}^{*}$ \\
\hline 7 & $x_{31}$ & $-x_{32}^{*}$ & $x_{29}$ & & $-x_{30}^{*}$ & $x_{79}$ & $-x_{56}^{*}$ & $\left|x_{77}\right|$ & \begin{tabular}{|l|l|}
$-x_{78}^{*}$ & $x_{127}$ \\
\end{tabular} & & \begin{tabular}{|l|}
$-x_{128}^{*}$ \\
\end{tabular} & $x_{125}$ & $-x_{126}^{*}$ \\
\hline 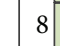 & $x_{35}$ & $-x_{36}^{*}$ & $x_{33}$ & & $-x_{34}^{*}$ & $x_{83}$ & $-x_{56}^{*}$ & $x_{81}$ & \begin{tabular}{|l|l|}
$-x_{82}^{*}$ & $x_{131}$ \\
\end{tabular} & & $-x_{132}^{*}$ & $x_{129}$ & $-x_{130}^{*}$ \\
\hline 9 & $x_{39}$ & $-x_{40}^{*}$ & $x_{37}$ & & $-x_{38}^{*}$ & $x_{87}$ & $-x_{52}^{*}$ & $x_{85}$ & $-x_{86}^{*} x_{135}$ & & $-x_{136}^{*}$ & $x_{133}$ & $-x_{134}^{*}$ \\
\hline 10 & $x_{43}$ & $-x_{44}^{*}$ & $x_{41} \mid F$ & $P_{A_{2} B_{2}}$ & $-x_{42}^{*}$ & $x_{91}$ & $-x_{56}^{*}$ & $x_{89}$ & \begin{tabular}{|l|l|}
$-x_{90}^{*}$ & $x_{139} I$ \\
\end{tabular} & $\overline{P_{A_{2} B}}$ & $-x_{140}^{*}$ & $x_{137}$ & $-x_{138}^{*}$ \\
\hline 11 & \begin{tabular}{|l|}
$x_{47}$ \\
\end{tabular} & $-x_{48}^{*}$ & $x_{45}$ & & $-x_{46}^{*}$ & $x_{95}$ & $-x_{96}^{*}$ & $x_{93}$ & \begin{tabular}{|l|l|}
$-x_{94}^{*}$ & $x_{143}$ \\
\end{tabular} & & $-x_{144}^{*}$ & $x_{141}$ & $-x_{142}^{*}$ \\
\hline & 0 & 1 & 2 & 3 & 4 & 5 & 6 & 7 & 8 & 10 & 11 & 12 & 13 \\
\hline
\end{tabular}

Branch $A_{1} B_{3}$

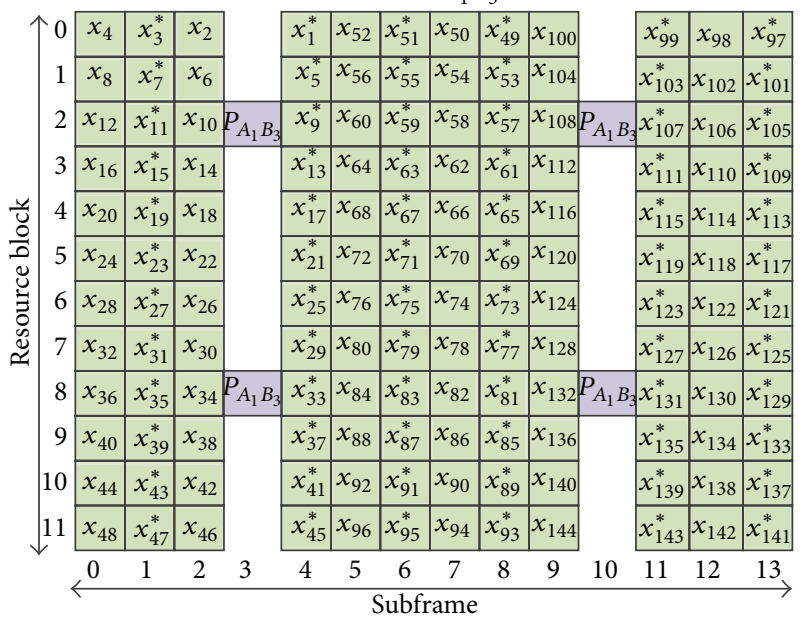

Branch $A_{2} B_{3}$

\begin{tabular}{|c|c|c|c|c|c|c|c|c|c|c|c|c|c|}
\hline \multirow{2}{*}{$\uparrow \begin{array}{c}0 \\
1\end{array}$} & $x_{4}$ & $x_{3}^{*}$ & $x_{2}$ & & \begin{tabular}{|l|}
$x_{1}^{*}$ \\
\end{tabular} & $x_{52}$ & $x_{51}^{*}$ & $x_{50}$ & \begin{tabular}{|l|l|}
$x_{49}^{*}$ & $x_{100}$ \\
\end{tabular} & & \begin{tabular}{|l|}
$x_{99}^{*}$ \\
\end{tabular} & $x_{98}$ & \\
\hline & $x_{8}$ & $x_{7}^{*}$ & $x$ & & $x_{5}^{*}$ & $x_{56}$ & $x_{55}^{*}$ & $x_{54}$ & \begin{tabular}{|l|l|}
$x_{53}^{*}$ & $x_{104}$ \\
\end{tabular} & & $x_{103}^{*}$ & $x_{102}$ & 10 \\
\hline \multirow{6}{*}{ 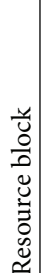 } & $x_{12}$ & $x_{11}^{*}$ & $x_{10}$ & & \begin{tabular}{l|l|}
$x_{9}^{*}$ \\
\end{tabular} & $x_{60}$ & $x_{59}^{*}$ & $x_{58}$ & \begin{tabular}{|l|l|}
$x_{57}^{*}$ & $x_{108}$ \\
\end{tabular} & & $x_{107}^{*}$ & 100 & \\
\hline & $x_{16}$ & $x_{15}^{*}$ & $x_{14}$ & & $x_{13}^{*}$ & $x_{64}$ & $x_{63}^{*}$ & $x_{62}$ & \begin{tabular}{|l|l|}
$x_{61}^{*}$ & $x_{112}$ \\
\end{tabular} & & $x_{111}^{*}$ & & \\
\hline & $x_{20}$ & $x_{19}^{*}$ & $x_{18}$ & & $x_{17}^{*}$ & $x_{68}$ & $x_{67}^{*}$ & $x_{66}$ & \begin{tabular}{|l|l|}
$x_{65}^{*}$ & $x_{116}$ \\
\end{tabular} & & $x_{115}^{*}$ & & $\varepsilon_{1}^{*}$ \\
\hline & $x_{24}$ & $x_{23}^{*}$ & $x_{22}$ & $P_{A_{2} B_{3}}$ & $x_{21}^{*}$ & $x_{72}$ & $x_{71}^{*}$ & $x_{70}$ & $x_{69}^{*}$ & & $x_{119}^{*}$ & & \\
\hline & $x_{28}$ & $x_{27}^{*}$ & $x_{26}$ & & $x_{25}^{*}$ & $x_{76}$ & $x_{75}^{*}$ & $x_{74}$ & $x_{73}^{*}{ }^{x}$ & & $x_{123}^{*}$ & 22 & \\
\hline & $x_{32}$ & $x_{31}^{*}$ & $x_{30}$ & & $x_{29}^{*}$ & $x_{80}$ & $x_{79}^{*}$ & $x_{78}$ & \begin{tabular}{|l|l|}
$x_{77}^{*}$ & $x_{128}$ \\
\end{tabular} & & $x_{127}^{*}$ & 126 & \\
\hline 8 & $x_{36}$ & $x_{35}^{*}$ & $x_{34}$ & & $x_{33}^{*}$ & $x_{84}$ & $x_{83}^{*}$ & $x_{82}$ & \begin{tabular}{|l|l|}
$x_{81}^{*}$ & $x_{132}$ \\
\end{tabular} & & $x_{131}^{*}$ & & $x$ \\
\hline (7) & $x_{40}$ & $x_{39}^{*}$ & $x_{38}$ & & $x_{37}^{*}$ & $x_{88}$ & $x_{87}^{*}$ & $x_{86}$ & \begin{tabular}{|l|l|}
$x_{85}^{*}$ & $x_{136}$ \\
\end{tabular} & & $x_{135}^{*}$ & 134 & $x_{13}^{*}$ \\
\hline 10 & $x_{44}$ & $x_{43}^{*}$ & $x_{42}$ & & $x_{41}^{*}$ & $x_{92}$ & $x_{91}^{*}$ & $x_{90}$ & \begin{tabular}{|l|l|}
$x_{89}^{*}$ & $x_{140}$ \\
\end{tabular} & & $x_{139}^{*}$ & $x_{138}$ & $x_{13}^{*}$ \\
\hline & $x_{48}$ & $x_{47}^{*}$ & $x_{46}$ & $P_{A_{2} B_{3}}$ & $x_{45}^{*}$ & $x_{96}$ & $x_{95}^{*}$ & $x_{94}$ & \begin{tabular}{|l|l|}
$x_{93}^{*}$ & $x_{144}$ \\
\end{tabular} & ${ }_{4} P_{A_{2} B_{3}}$ & $x_{143}^{*}$ & $x_{142}$ & $x_{14}^{*}$ \\
\hline & & 1 & 2 & 3 & 4 & 5 & 6 & 7 & 8 & 10 & 11 & 12 & 1 \\
\hline
\end{tabular}

(c) Data mapping at $\mathrm{Tx} A_{p} B_{3}$

FIgURE 10: Downlink data mapping of the LTE-A resource blocks used in the simulation. 


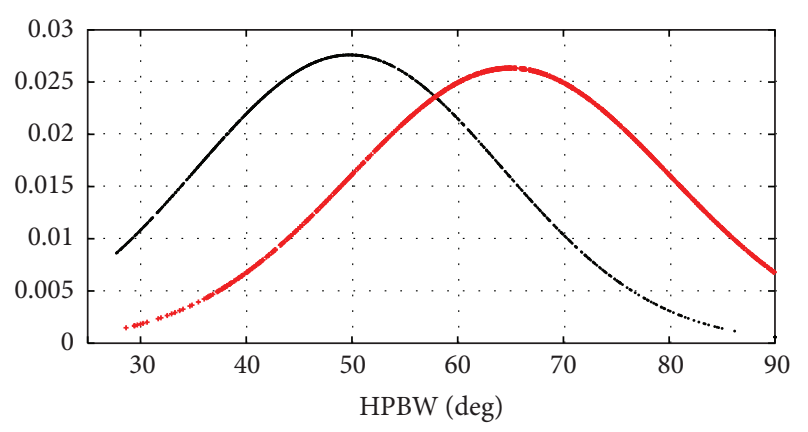

- With the proposed AE selection scheme

+ Without the proposed AE selection scheme

Figure 11: Probability density of HPBW.

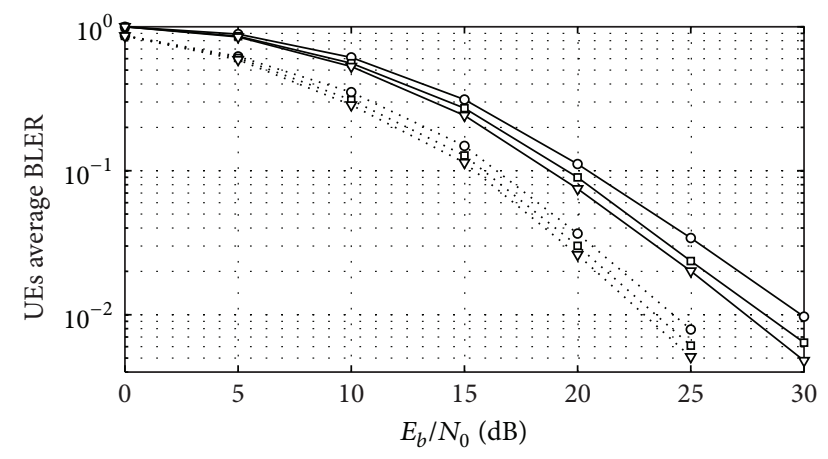

- - Case 1, PACE, outdoor, NLOS, $3 \mathrm{~km} / \mathrm{h}$

$\rightarrow$ Case 2, PACE, outdoor, NLOS, $3 \mathrm{~km} / \mathrm{h}$

$\rightarrow$ Case 3, PACE, outdoor, NLOS, $3 \mathrm{~km} / \mathrm{h}$

. o. Case 1, PACE, outdoor, LOS, $3 \mathrm{~km} / \mathrm{h}$

a.. Case 2, PACE, outdoor, LOS, $3 \mathrm{~km} / \mathrm{h}$

$\cdot \nabla \cdot$ Case 3, PACE, outdoor, LOS, $3 \mathrm{~km} / \mathrm{h}$

FIGURE 12: Users average BLER performances based on the proposed diversity system architecture.

Figure 12 compares the users' average block error rate (BLER) performances obtained, based on the proposed diversity system architecture by using different transmission schemes, including Case 1 without STBC and TxBF, Case 2 with STBC only, and Case 3 with STBC and TxBF via the LTE pilot-assistant practical channel estimation (PACE) approach. According to Figure 12, Case 1 has the worst performance because there is no space-time coding and $\mathrm{BF}$ gain achieved. About $1.7 \mathrm{~dB}$ of signal-to-noise ratio (SNR) gain at the target BLER can be achieved by Case 2, compared with Case 1 under the non-line-of-sight NLOS scenario. There is about a $1 \mathrm{~dB}$ SNR gain that can be further achieved by employing the TxBF of Case 3 . In addition, the simulation results under the LOS scenario are also provided as a comparison, where almost $7 \mathrm{~dB}$ gain can be obtained when the LOS exists for those three cases.

Next, we simulate the BCE approaches and compare its efficiency with the PACE based on the proposed diversity system architecture. Figure 13 demonstrates the users' average BLER via PACE and $\mathrm{BCE}$ approaches for three different
TABLE 1: Simulation parameters.

\begin{tabular}{|c|c|}
\hline Parameters & Value \\
\hline Carrier frequency & $1.8 \mathrm{GHz}$ \\
\hline System bandwidth & $20 \mathrm{MHz}$ \\
\hline FFT size & 2048 \\
\hline Number of data carriers & 1200 \\
\hline Number of samples in CP & 144 \\
\hline Subcarrier spacing & $15 \mathrm{kHz}$ \\
\hline $\begin{array}{l}\text { BS antenna configuration } \\
\text { (PM-MIMO) }\end{array}$ & $1 \mathrm{MUX} \times 32 \mathrm{AE} \times 3$ branches \\
\hline BS AE spacing & Half wavelength \\
\hline User's antenna configuration & $1 \mathrm{AE} \times 3$ branches \\
\hline \multirow{2}{*}{ Number of users } & 4 for 3D-BF diversity \\
\hline & 12 for 3D-BF multiplexing \\
\hline Radius of user's local area $\left(r_{\mathrm{UE}}\right)$ & $15 \mathrm{~m}$ \\
\hline Antenna type & Dipole \\
\hline Modulation & QPSK \\
\hline Number of scatterers & $\begin{array}{l}\text { Outdoor: } 4 \text { clusters with } 16 \\
\text { scatterers per cluster ( } 64 \text { in } \\
\text { total) }\end{array}$ \\
\hline Scattering sphere radius & $10 \mathrm{~m}$ \\
\hline Velocity of UEs & 3,60 , and $120 \mathrm{~km} / \mathrm{h}$ \\
\hline Fading & Flat \\
\hline XPD value & $\begin{array}{c}5.8 \mathrm{~dB} \text { and } 9.7 \mathrm{~dB} \text { for NLOS } \\
\text { and LOS }\end{array}$ \\
\hline Correlation & $\begin{array}{c}0.32 \text { and } 0.34 \text { for NLOS and } \\
\text { LOS }\end{array}$ \\
\hline Rician $K$-factor & $9 \mathrm{~dB}$ \\
\hline
\end{tabular}

user's velocities, including 3, 60, and $120 \mathrm{~km} / \mathrm{h}$, under the NLOS scenario. An extension of other researches $[14,31]$ is applied to simulations of the polarized MIMO channel, for which channel characteristics are also listed in Table 1. We see that the PACE performance decreases a lot due to high mobility at 60 and $120 \mathrm{~km} / \mathrm{h}$, which indicates that the number of pilots is not enough to compensate the channel correctly in an environment with fast time-varying phase response. The pilot density is $14.3 \%$ for PACE scheme, and by considering the trade-off regarding spectrum efficiency, BCE that requires no, or a minimal number of, pilots might be better employed. Figure 13 additionally shows the performances of BCE schemes where performance is found that is not relevant to the user's velocity. Compared with the BCE reported by Ngo and Larsson [29], our proposed BCE performance is better because the EVD is based on low correlated submatrix and has less complexity for doing the EVD based on a submatrix with a smaller size. Due to no pilot contamination by $\mathrm{BCE}$ approach, the proposed $\mathrm{BCE}$ outperforms PACE for a velocity of $3 \mathrm{~km} / \mathrm{h}$ when $E_{b} / N_{0}$ is less than $25 \mathrm{~dB}$. However, BCE has a higher complexity than PACE, and it is condition constraint. Again, according to Figure 13, BCE performs worse than PACE with a velocity of $3 \mathrm{~km} / \mathrm{h}$ when $E_{b} / N_{0}$ is larger than $25 \mathrm{~dB}$, and the trend of error floor for 


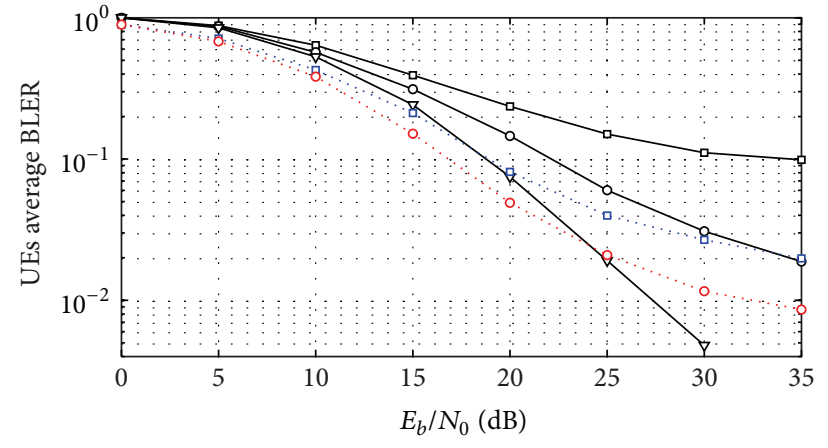

$\rightarrow$ PACE, outdoor, NLOS, $120 \mathrm{~km} / \mathrm{h}$

$\multimap$ PACE, outdoor, NLOS, $60 \mathrm{~km} / \mathrm{h}$

$\rightarrow$ PACE, outdoor, NLOS, $3 \mathrm{~km} / \mathrm{h}$

… BCE by Ngo and Larsson, outdoor, NLOS, $3 / 60 / 120 \mathrm{~km} / \mathrm{h}$

ㅇ. Proposed BCE, outdoor, NLOS, 3/60/120 km/h

FIGURE 13: Users' average BLER performance by PACE and BCE under an NLOS environment based on the proposed diversity system architecture.

the proposed BCE occurs at $30 \mathrm{~dB}$. Figure 14 is given by considering the signal LOS component, where performance characteristics observed are the same as Figure 13.

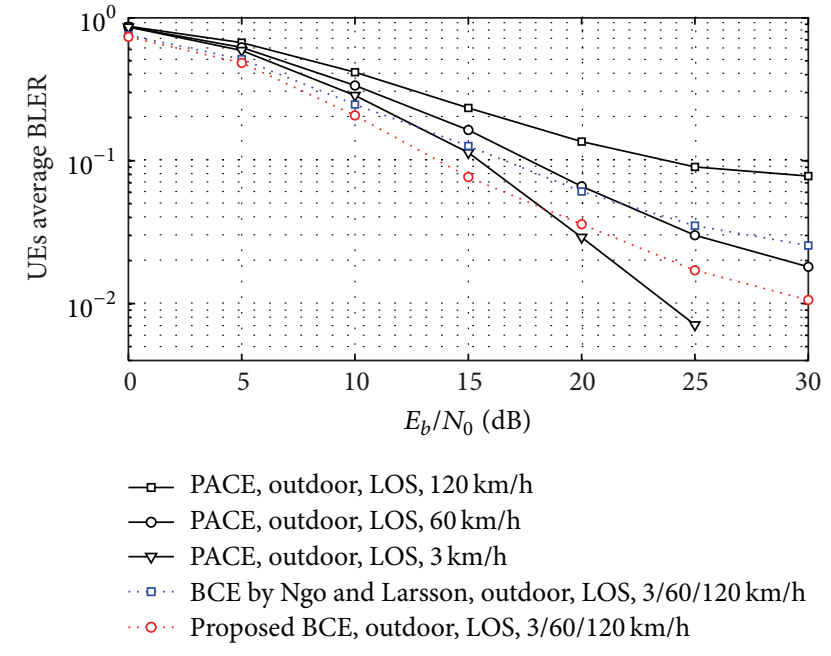

FIGURE 14: Users' average BLER performance by PACE and BCE under a LOS environment based on the proposed diversity system architecture.

At last, we simulate the proposed multiplexing system architecture via 3D beams, where the channel matrix dedicated to a user is then given as

$$
\mathbf{h}_{T A_{p}, k}(t)=\sqrt{\frac{\eta}{S}} \sum_{s=1}\left(\begin{array}{cccc}
h_{T A_{1} B_{b}, k_{B_{1}}}(t) & \sqrt{\mu} \cdot h_{T A_{1} B_{b}, k_{B_{2}}}(t) & \sqrt{\mu} \cdot h_{T A_{1} B_{b}, k_{B_{3}}}(t) \\
h_{T A_{2} B_{b}, k_{B_{1}}}(t) & \sqrt{\mu} \cdot h_{T A_{2} B_{b}, k_{B_{2}}}(t) & \sqrt{\mu} \cdot h_{T A_{2} B_{b}, k_{B_{3}}}(t) \\
\vdots & \vdots & \vdots \\
h_{T A_{p} B_{b}, k_{B_{1}}}(t) & \sqrt{\mu} \cdot h_{T A_{p} B_{b}, k_{B_{2}}}(t) & \sqrt{\mu} \cdot h_{T A_{p} B_{b}, k_{B_{3}}}(t)
\end{array}\right) \text {. }
$$

According to channel matrix (30), the performance of the EVD-based BCE approach may decrease because the constraint conditions of $\mathrm{BCE}$ are not preserved. However, the pilot contamination effect is reduced because there are fewer antennae using the same pilot positions. For example, there are $24 \mathrm{Tx}$ antennae used in the proposed diversity scheme dedicated to a user, where four antennae share the same pilot positions. In the proposed multiplexing scheme, eight Tx antennae are dedicated to a user, where two of them need to share the same pilot position with other antennae. Figures 15 and 16 illustrate the user's average BLER comparing the multiplexing and diversity system architectures by $3 \mathrm{D}$ beams. Note that the data rate of multiplexing is three times higher than the diversity scheme. For PACE performances, there is about $3 \mathrm{~dB}$ SNR gain at target BLER of $10^{-2}$ (at $3 \mathrm{~km} / \mathrm{h}$ case) achieved by the diversity scheme, compared with the multiplexing scheme, mainly due to the gain of QO-STBC. However, the gain is not significant, because the pilot contamination effect is reduced when employing the multiplexing scheme with fewer antennae using the same pilot positions. The BCE performance, as discussed earlier, cannot be maintained with multiplexing since the constraint condition of $\mathrm{BCE}$ is not fulfilled.

\section{Conclusions}

M-MIMO has been developed as a promising technology due to several attractive features. However, there is less research on M-MIMO systems with antenna polarization, where the antenna polarization can cope with one of crucial constraints: a dimension in space to implement the M-MIMO. In this paper, we propose a PM-MIMO array system with three orthogonally colocated antenna branches equipped at each $\mathrm{AE}$ of an M-MIMO system. System architectures of diversity and multiplexing schemes realized by polarized 3D beams are then proposed based on the proposed PM-MIMO array system. An array selection scheme for 3D-BF applications is additionally provided in this paper to efficiently optimize the beam-width and to enhance system performance by the exploration of diversity and multiplexing gains. In order to avoid pilot contamination in PM-MIMO, we also propose a $\mathrm{BCE}$ approach to exploit pairwise orthogonality according to the particular characteristics of PM-MIMO systems. With the proposed BCE approach, $14.3 \%$ of spectral efficiency can be increased, while the gain in BLER performance is dependent on mobility, compared with PACE. Finally, the simulation results, including the performances comparison 


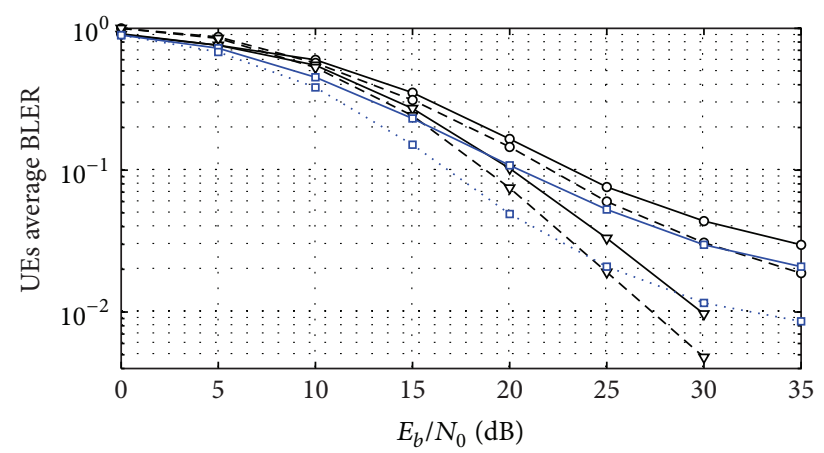

$\multimap$ 3D-BF multiplexing, PACE, outdoor, NLOS, $60 \mathrm{~km} / \mathrm{h}$ - o- 3D-BF diversity, PACE, outdoor, NLOS, $60 \mathrm{~km} / \mathrm{h}$

$\rightarrow-3 \mathrm{D}$-BF multiplexing, PACE, outdoor, NLOS, $3 \mathrm{~km} / \mathrm{h}$

$-\nabla-3 \mathrm{D}-\mathrm{BF}$ diversity, PACE, outdoor, NLOS, $3 \mathrm{~km} / \mathrm{h}$

$\neg-3 \mathrm{D}-\mathrm{BF}$ multiplexing, BCE, outdoor, NLOS, $3 / 60 \mathrm{~km} / \mathrm{h}$

a. 3D-BF diversity, BCE, outdoor, NLOS, $3 / 60 \mathrm{~km} / \mathrm{h}$

FIGURE 15: Users' average BLER performance by the proposed diversity and multiplexing schemes via $3 \mathrm{D}$ beams under an NLOS environment.

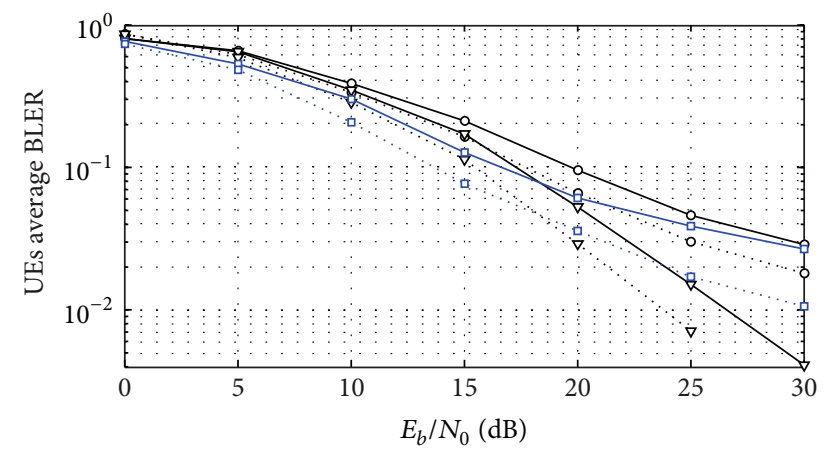

-o- 3D-BF multiplexing, PACE, outdoor, LOS, $60 \mathrm{~km} / \mathrm{h}$

o.. 3D-BF diversity, PACE, outdoor, LOS, $60 \mathrm{~km} / \mathrm{h}$

$\rightarrow-3 \mathrm{D}$-BF multiplexing, PACE, outdoor, LOS, $3 \mathrm{~km} / \mathrm{h}$

$\cdot \nabla \cdot 3 \mathrm{D}-\mathrm{BF}$ diversity, PACE, outdoor, LOS, $3 \mathrm{~km} / \mathrm{h}$

$\rightarrow-3 \mathrm{D}$-BF multiplexing, BCE, outdoor, LOS, $3 / 60 \mathrm{~km} / \mathrm{h}$

- . 3D-BF diversity, BCE, outdoor, LOS, $3 / 60 \mathrm{~km} / \mathrm{h}$

FIGURE 16: Users average BLER performance by the proposed diversity and multiplexing schemes via 3D beams under a LOS environment.

between PACE and BCE, as well as diversity and multiplexing schemes, confirmed the validity of our proposals.

\section{Conflict of Interests}

The authors declare that there is no conflict of interests regarding the publication of this paper.

\section{Acknowledgments}

This research was supported by the MSIP (Ministry of Science, ICT and Future Planning), Korea, under the ITRC (Information Technology Research Center) Support Program (IITP-2015-H8501-15-1019) supervised by the IITP (Institute for Information \& Communications Technology Promotion) and also was supported by "the Fundamental Research Funds for the Central Universities (2015B30614).”

\section{References}

[1] T. L. Marzetta, "Noncooperative cellular wireless with unlimited numbers of base station antennas," IEEE Transactions on Wireless Communications, vol. 9, no. 11, pp. 3590-3600, 2010.

[2] D. W. K. Ng, E. S. Lo, and R. Schober, "Energy-efficient resource allocation in OFDMA systems with large numbers of base station antennas," IEEE Transactions on Wireless Communications, vol. 11, no. 9, pp. 3292-3304, 2012.

[3] J. Hoydis, S. Ten Brink, and M. Debbah, "Massive MIMO in the UL/DL of cellular networks: how many antennas do we need?" IEEE Journal on Selected Areas in Communications, vol. 31, no. 2, pp. 160-171, 2013.

[4] J. G. Andrews, H. Claussen, M. Dohler, S. Rangan, and M. C. Reed, "Femtocells: past, present, and future," IEEE Journal on Selected Areas in Communications, vol. 30, no. 3, pp. 497-508, 2012.

[5] H. Q. Ngo, E. G. Larsson, and T. L. Marzetta, "Energy and spectral efficiency of very large multiuser MIMO systems," IEEE Transactions on Communications, vol. 61, no. 4, pp. 1436-1449, 2013.

[6] A. Fehske, G. Fettweis, J. Malmodin, and G. Biczok, “The global footprint of mobile communications: the ecological and economic perspective," IEEE Communications Magazine, vol. 49, no. 8, pp. 55-62, 2011.

[7] T. L. Marzetta, "How much training is required for multiuser MIMO?" in Proceedings of the 40th Asilomar Conference on Signals, Systems, and Computers (ACSSC '06), pp. 359-363, Pacific Grove, Calif, USA, November 2006.

[8] N. Krishnan, R. D. Yates, and N. B. Mandayam, "Uplink linear receivers for multi-cell multiuser MIMO with pilot contamination: large system analysis," IEEE Transactions on Wireless Communications, vol. 13, no. 8, pp. 4360-4373, 2014.

[9] H. Q. Ngo, T. L. Marzetta, and E. G. Larsson, "Analysis of the pilot contamination effect in very large multicell multiuser MIMO systems for physical channel models," in Proceedings of the 36th IEEE International Conference on Acoustics, Speech, and Signal Processing (ICASSP '11), pp. 3464-3467, IEEE, Prague, Czech Republic, May 2011.

[10] S. K. Mohammed and E. G. Larsson, "Single-user beamforming in large-scale MISO systems with per-antenna constantenvelope constraints: the doughnut channel," IEEE Transactions on Wireless Communications, vol. 11, no. 11, pp. 3992-4005, 2012.

[11] X. Gao, O. Edfors, F. Rusek, and F. Tufvesson, "Linear pre-coding performance in measured very-large MIMO channels," in Proceedings of the IEEE Vehicular Technology Conference (VTC Fall '11), pp. 1-5, San Francisco, Calif, USA, September 2011.

[12] S. Payami and F. Tufvesson, "Channel measurements and analysis for very large array systems at $2.6 \mathrm{GHz}$," in Proceedings of the 6th European Conference on Antennas and Propagation (EuCAP '12), pp. 433-437, IEEE, Prague Czech Republic, March 2012.

[13] J. Hoydis, C. Hoek, T. Wild, and S. T. Brink, "Channel measurements for large antenna arrays," in Proceedings of the 9th International Symposium on Wireless Communication Systems (ISWCS '12), pp. 811-815, IEEE, Paris, France, August 2012. 
[14] X. Su, B. Hui, and K. H. Chang, "3-D MIMO channel modeling with beamforming analysis for dual-polarized antenna systems," in Proceedings of the IEEE 78th Vehicular Technology Conference (VTC Fall '13), pp. 1-5, Las Vegas, Nev, USA, September 2013.

[15] A. S. Y. Poon and D. N. C. Tse, "Degree-of-freedom gain from using polarimetric antenna elements," IEEE Transactions on Information Theory, vol. 57, no. 9, pp. 5695-5709, 2011.

[16] X. Su and K. H. Chang, "Polarized uniform linear array system: beam radiation pattern, beamforming diversity order, and channel capacity," International Journal of Antenna and Propagation, vol. 2015, Article ID 371236, 9 pages, 2015.

[17] M.-T. Dao, V.-A. Nguyen, Y.-T. Im, S.-O. Park, and G. Yoon, "3D polarized channel modeling and performance comparison of MIMO antenna configurations with different polarizations," IEEE Transactions on Antennas and Propagation, vol. 59, no. 7, pp. 2672-2682, 2011.

[18] X. Su and K. Chang, "A comparative study on wireless backhaul solutions for beyond 4G network," in Proceedings of the 27th International Conference on Information Networking (ICOIN '13), pp. 505-510, IEEE, Bangkok, Thailand, January 2013.

[19] Y. Li, X. D. Ji, D. Liang, and Y. Li, "Dynamic beamforming for three-dimensional MIMO technique in LTE-advanced networks," International Journal of Antennas and Propagation, vol. 2013, Article ID 764507, 8 pages, 2013.

[20] J. Li, U. Park, and S. Kim, "An efficient rate one STBC scheme with 3 transmit antennas," in Proceedings of the International Conference on Wireless Communications, Networking and Mobile Computing (WiCOM '08), pp. 1-4, IEEE, Dalian, China, October 2008.

[21] U. Park, S. Kim, K. Lim, and J. Li, "A novel QO-STBC scheme with linear decoding for three and four transmit antennas," IEEE Communications Letters, vol. 12, no. 12, pp. 868-870, 2008.

[22] W. Liu, "Adaptive wideband beamforming with sensor delaylines," Signal Processing, vol. 89, no. 5, pp. 876-882, 2009.

[23] H. F. Yin, D. Gesbert, M. C. Filippou, and Y. Z. Liu, "Decontaminating pilots in massive MIMO systems," in Proceedings of the IEEE International Conference on Communications (ICC '13), pp. 3170-3175, IEEE, Budapest, Hungary, June 2013.

[24] L. Lu, G. Y. Li, A. L. Swindlehurst, A. Ashikhmin, and R. Zhang, "An overview of massive MIMO: benefits and challenges," IEEE Journal on Selected Topics in Signal Processing, vol. 8, no. 5, pp. 742-758, 2014.

[25] T. E. Bogale and L. B. Le, "Pilot optimization and channel estimation for multiuser massive MIMO systems," in Proceedings of the 48th Annual Conference on Information Sciences and Systems (CISS '14), pp. 1-6, Princeton, NJ, USA, March 2014.

[26] J. Jose, A. Ashikhmin, T. L. Marzetta, and S. Vishwanath, "Pilot contamination and precoding in multi-cell TDD systems," IEEE Transactions on Wireless Communications, vol. 10, no. 8, pp. 2640-2651, 2011.

[27] E. Beres and R. Adve, "Blind channel estimation for orthogonal STBC in MISO systems," IEEE Transactions on Vehicular Technology, vol. 56, no. 4, pp. 2042-2050, 2007.

[28] B. Muquet, M. de Courville, and P. Duhamel, "Subspace-based blind and semi-blind channel estimation for OFDM systems," IEEE Transactions on Signal Processing, vol. 50, no. 7, pp. 16991712, 2002.
[29] H. Q. Ngo and E. G. Larsson, "EVD-based channel estimation in multicell multiuser MIMO systems with very large antenna arrays," in Proceedings of the IEEE International Conference on Acoustics, Speech, and Signal Processing (ICASSP '12), pp. 32493252, IEEE, Kyoto, Japan, March 2012.

[30] MIMO transmission schemes for LTE and HSPA networks, 2009, http://persons.unik.no/porten/teaching/UNIK4180/Materiell/Mimo_Transmission_Schemes_for_LTE_and_HSPA_Networks_June-2009.pdf.

[31] K. Jeon, X. Su, B. Hui, and K. Chang, "Practical and simple wireless channel models for use in multipolarized antenna systems," International Journal of Antennas and Propagation, vol. 2014, Article ID 619304, 10 pages, 2014.

[32] ETSI TS 136211 v10.2.0 (2011-06), "LTE evolved universal terrestrial radio access (E-UTRA), physical channels and modulation," Technical Specification, 2011. 

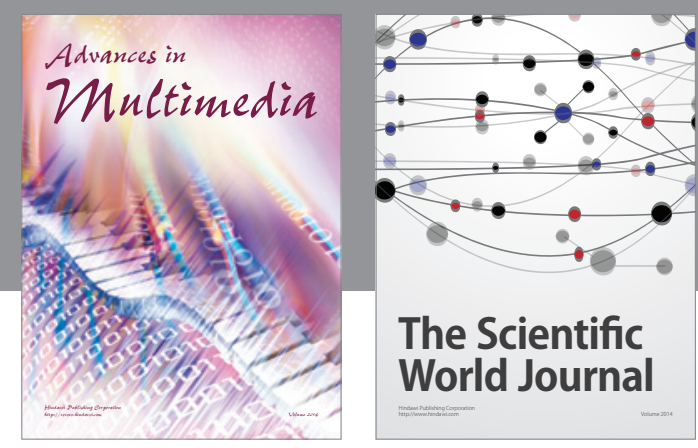

The Scientific World Journal
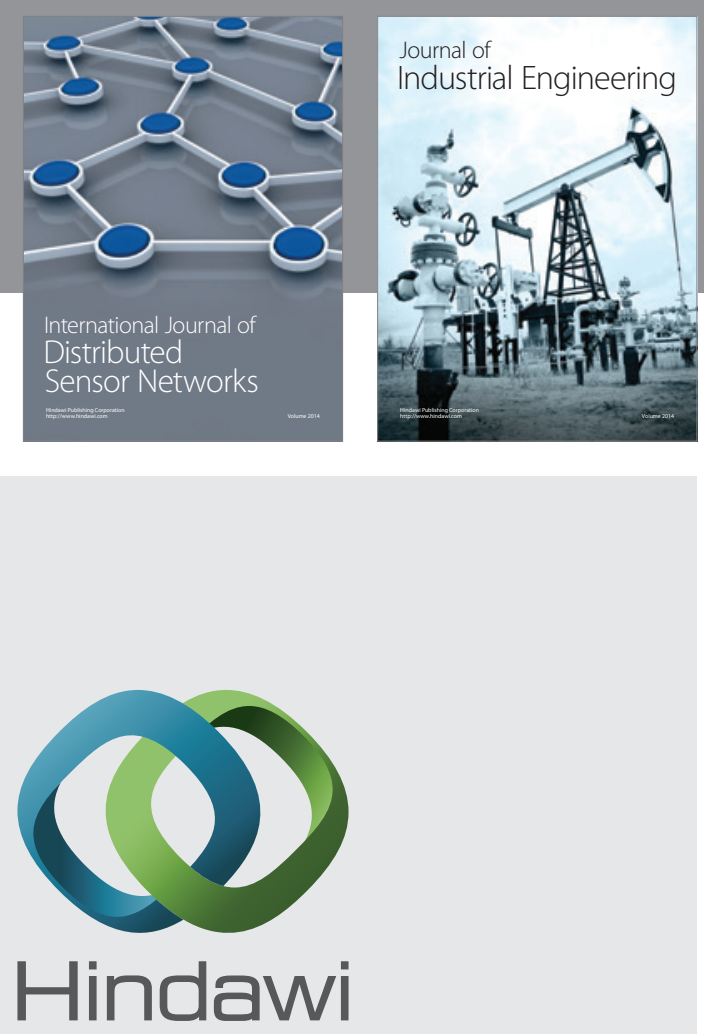

Submit your manuscripts at

http://www.hindawi.com

\section{Computer Networks} and Communications
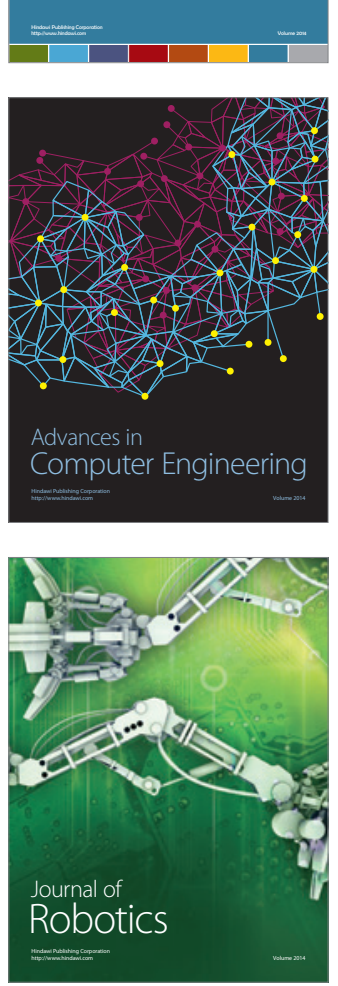
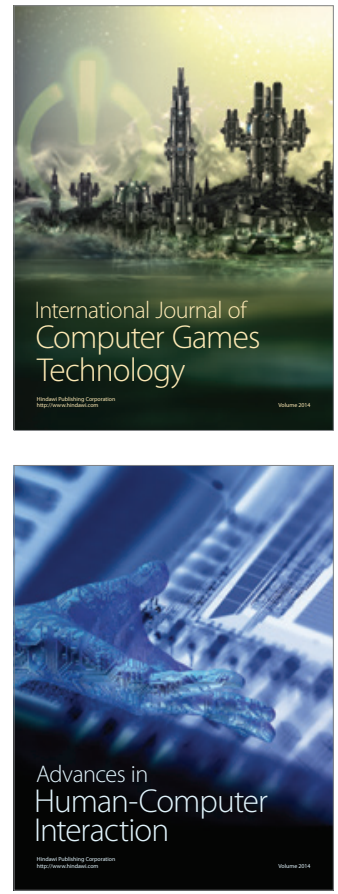
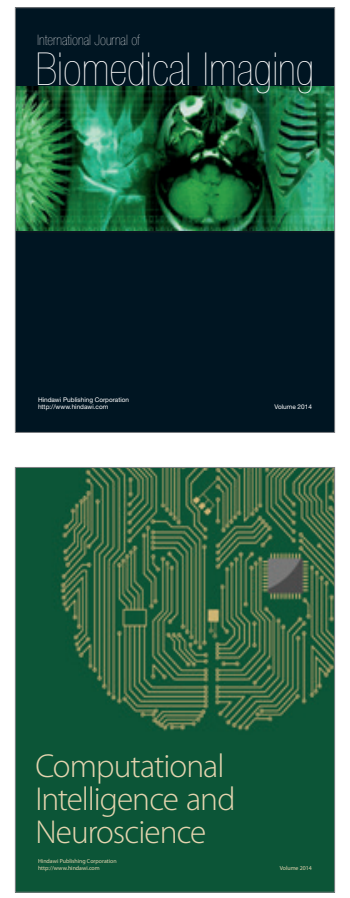
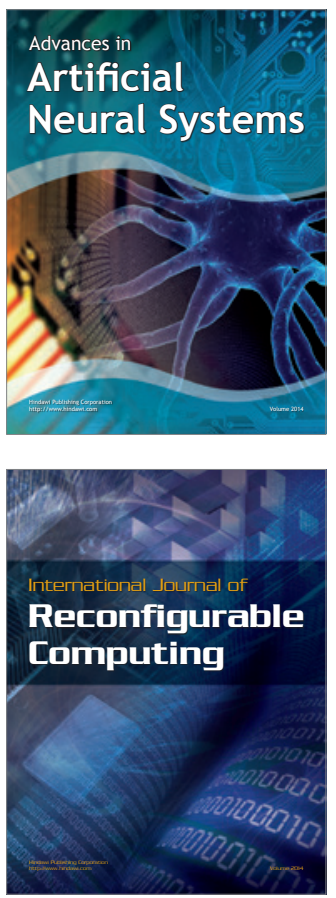
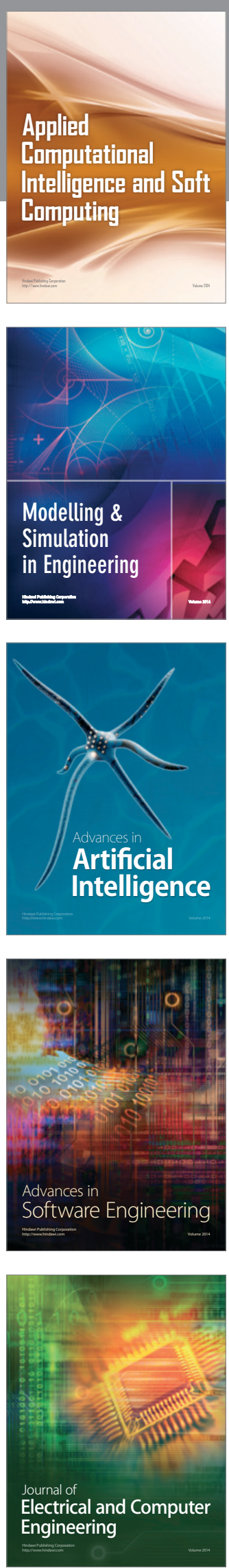